\title{
əThe Role of Curvature in Modifying Frontal Instabilities. Part I: Review of Theory and Presentation of a Nondimensional Instability Criterion
}

\author{
Christian E. Buckingham, ${ }^{\mathrm{a}, \mathrm{b}}$ Jonathan Gula, ${ }^{\mathrm{a}}$ AND XAvier CARTOn ${ }^{\mathrm{a}}$ \\ ${ }^{a}$ Université de Bretagne Occidentale, CNRS, IRD, Ifremer, Laboratoire d'Océanographie Physique et Spatiale, \\ IUEM, Plouzané, France \\ ${ }^{\mathrm{b}}$ British Antarctic Survey, Cambridge, United Kingdom
}

(Manuscript received 4 November 2019, in final form 6 October 2020)

\begin{abstract}
In this study, we examine the role of curvature in modifying frontal stability. We first evaluate the classical criterion that the Coriolis parameter $f$ multiplied by the Ertel potential vorticity (PV) $q$ is positive for stable flow and that instability is possible when this quantity is negative. The first portion of this statement can be deduced from Ertel's PV theorem, assuming an initially positive $f q$. Moreover, the full statement is implicit in the governing equation for the mean geostrophic flow, as the discriminant, $f q$, changes sign. However, for curved fronts in cyclogeostrophic or gradient wind balance (GWB), an additional term enters the discriminant owing to conservation of absolute angular momentum $L$. The resulting expression, $(1+$ $\mathrm{Cu}) f q<0$ or $L q<0$, where $\mathrm{Cu}$ is a nondimensional number quantifying the curvature of the flow, simultaneously generalizes Rayleigh's criterion by accounting for baroclinicity and Hoskins's criterion by accounting for centrifugal effects. In particular, changes in the front's vertical shear and stratification owing to curvature tilt the absolute vorticity vector away from its thermal wind state; in an effort to conserve the product of absolute angular momentum and Ertel PV, this modifies gradient Rossby and Richardson numbers permitted for stable flow. This forms the basis of a nondimensional expression that is valid for inviscid, curved fronts on the $f$ plane, which can be used to classify frontal instabilities. In conclusion, the classical criterion $f q<0$ should be replaced by the more general criterion for studies involving gravitational, centrifugal, and symmetric instabilities at curved density fronts. In Part II of the study, we examine interesting outcomes of the criterion applied to low-Richardson-number fronts and vortices in GWB.
\end{abstract}

SIGNIFICANCE STATEMENT: Considerable progress has been made by considering ocean fronts to be in geostrophic balance. By this, we mean that fluid parcels accelerate as a result of horizontal pressure gradients and Earth's rotation. A good example of this is in our efforts to understand symmetric instability, a process thought to impact energy, buoyancy, and tracer budgets in the ocean. However, we wanted to know how the physics might change if we accounted for centrifugal forces, or curvature. It turns out that this same question had been asked and answered nearly 100 years ago. However, the new criteria that we introduce in Part I yield (in Part II) one result that is new: in low-stratified waters, curved cyclonic fronts become strongly unstable and curved anticyclonic fronts become marginally stable. This suggests that highly curved cyclonic fronts and vortices are symmetrically unstable, with potential implications for the aforementioned budgets.

KEYWORDS: Instability; Ocean dynamics; Potential vorticity; Turbulence; Frontogenesis/frontolysis; Fronts; Vortices; Angular momentum

\section{Introduction}

There has been considerable interest in submesoscale dynamics in the ocean in recent years. Nominally characterized by horizontal scales of $0.1-10 \mathrm{~km}$ and temporal scales of hours to days, this regime is host to a number of instabilities not present within the quasigeostrophic (QG) regime. In this dynamical framework, horizontal and vertical shears are enhanced to such an extent that one observes an increase in frontal instabilities (Thomas et al. 2008; McWilliams 2016). These include baroclinic instability reminiscent of Charneyand Eady-like instabilities (Charney 1947; Eady 1949), but also frontal instabilities that occur when the Ertel potential

\footnotetext{
D Denotes content that is immediately available upon publication as open access.
}

Corresponding author: Christian E. Buckingham, christian. buckingham@univ-brest.fr vorticity (PV) becomes opposite in sign to the background QG PV, subsequently leading to turbulence and mixing.

This elevated interest in submesoscale dynamics has been prompted by an increased ability to resolve finescale horizontal and vertical gradients in observations, for example, from autonomous float/glider measurements when combined with satellite measurements. At the same time, there has been a marked increase in computational capability, such that modeling frontal flows at hundred-meter scales is now possible and will soon become routine. Important to the oceanographer and climate scientist is the potential of such small-scale dynamics to modify large-scale dynamics, including 1) ocean circulation, 2) ocean-atmosphere heat exchange, and 3) tracer exchange between the deep and upper oceans.

Interpreting dynamics at these fine spatial and temporal scales, however, poses a significant challenge. While Earth's rotation plays an important role in the horizontal momentum equations, it is largely acknowledged that strong mean flows and enhanced shear can cause advective terms to be of equal order at the submesoscale. Thus, simply assuming a geostrophic 
balance-that is, a balance between horizontal pressure gradient and Coriolis terms - may not be appropriate. Despite this, it must be acknowledged that considerable progress has been made in our understanding of dynamics at these fine scales by considering the mean state to be in thermal wind balance (TWB) and the perturbations themselves to be ageostrophic. One example where this is perhaps most true is in better understanding a phenomenon known as symmetric instability, and sometimes referred to as generalized inertial instability, slant-wise convection, or simply centrifugal instability (Hoskins 1974; Thorpe and Rotunno 1989; Thomas et al. 2008; Taylor and Ferrari 2009).

\section{a. Background: Generalized inertial instability or symmetric instability}

Symmetric instability is a vertical shear instability found within baroclinic fronts. Though the flow may be neither gravitationally unstable nor inertially unstable, within a front it can still be unstable to small disturbances in a direction inclined to the horizontal, where disturbances typically take the form of internal waves (Mooers 1975; Li et al. 2019). An understanding of this phenomenon largely grew out of early investigations of the stability of atmospheric vortices. While scientifically and mathematically fascinating in their own right, such investigations had relevance for weather prediction in the atmosphere and were therefore of practical importance, as well.

Notable contributions were made by several scientists, including Solberg (1936), Fjortoft (1944, 1950), Eliassen (1951), Ooyama (1966), and Yanai and Tokioka (1969). Solberg (1936) employed ring-displacement arguments of Rayleigh (1917) and derived a stability criterion valid for axisymmetric baroclinic vortices. Fjortoft (1950) also derived a necessary criterion for stability of a vortex. Because his work employed energetic arguments, the sufficiency of the criterion is often attributed to Fjortoft (1950). Both scientists' contributions are summarized in work by Eliassen and Kleinschmidt (1957) and van Mieghem et al. (1951).

Ooyama (1966) later expanded on these efforts, demonstrating that Fjortoft's criterion for vortex stability also served to define the marginally stable state and was, therefore, a necessary and sufficient criterion for instability. To do this, Ooyama (1966) formulated an analytical initial value problem (IVP), integrated the kinetic energy (KE) equation, and demonstrated that instability occurs if at least one set of initial conditions results in unbounded growth of total KE. In turn, motivated by Ooyama's work, Yanai and Tokioka (1969) constructed a numerical IVP, integrated the equations of motion for a vortex, and obtained an instability characterized by motion in the meridional plane-that is, in radial and vertical directions. Because of the azimuthal symmetry of parcel motion, the instability is now known as symmetric instability though it largely resembles classical inertial or centrifugal instability in appearance (Kloosterziel et al. 2007, their Fig. 1).

The stability criterion for symmetric instability within axisymmetric vortices, first given by Solberg (1936) and proved sufficient by Fjortoft (1950) and Ooyama (1966), can be summarized as follows. Instability is possible in an inviscid, baroclinic vortex when

$$
\Phi=[(f+2 \bar{v} / r)(f+\bar{\zeta})]_{\theta}<0
$$

where the quantity in brackets is evaluated along isentropic surfaces, $f$ is the Coriolis parameter or planetary vorticity, $\bar{v}$ is the mean azimuthal velocity, $r$ is the radius, and $\bar{\zeta}=(1 / r) \partial_{r}(r \bar{v})$ is the vertical component of mean relative vorticity. Since the bracketed quantity corresponds to the Rayleigh discriminant ${ }^{1}$ for inertial instability of barotropic vortices (Chandrasekhar 1961; Kloosterziel and van Heijst 1991; Mutabazi et al. 1992), one can equivalently view symmetric instability as an "inertial instability on isentropes." Equation (1) is therefore a generalized Rayleigh criterion for baroclinic vortices. Astrophysicists also use this criterion in studies pertaining to the stability of accretion disks where it is better known as Solberg's criterion (Nelson et al. 2013; Rüdiger et al. 2002). Finally, note that in the oceanic context, Eq. (1) can be evaluated approximately along isopycnals or, in the deep ocean, on neutral density surfaces (Jackett and McDougall 1997).

\section{b. Connection to Ertel's $P V$}

It was not until Hoskins (1974) that an explicit connection between the criterion for symmetric instability and the Ertel PV was made. Of particular note, Hoskins (1974) employed the TWB model of Ooyama [1966, their Eq. (19)] and showed that the criterion for instability can be restated as follows. In the Northern Hemisphere, symmetric instability is possible within a front in $\mathrm{TWB}^{2}$ when $q<0$, where

$$
q=\omega_{a} \cdot \nabla b
$$

is the Ertel PV (Ertel 1942). In this expression, $\boldsymbol{\omega}_{a}=\mathbf{2} \boldsymbol{\Omega}+$ $\nabla \times \mathbf{u} \approx f \hat{\mathbf{z}}+\nabla \times \mathbf{u}$ is the absolute vorticity, $\nabla \times \mathbf{u}$ is relative vorticity, and $f=2|\boldsymbol{\Omega}| \sin \theta$ is the vertical component of planetary vorticity at latitude $\theta$. Moreover, $b=-g \rho / \rho_{o}$ is buoyancy, $g$ is acceleration due to gravity, $\rho$ is density, and $\rho_{o}$ is a reference density. Multiplying by the Coriolis parameter to eliminate the hemispheric dependence, assuming stable stratification and restricting analysis away from the equator, the instability criterion can be recast in nondimensional form:

$$
q^{\prime}=1+\mathrm{Ro}-\mathrm{Ri}^{-1}<0
$$

In this expression, Ro $=\bar{\zeta} / f$ is the gradient Rossby number, $\mathrm{Ri}=N^{2} /\left|\partial_{z} \mathbf{u}\right|^{2}$ is the gradient Richardson number, $N^{2}=\partial_{z} \bar{b}$ is the vertical stratification, and $\partial_{z} \mathbf{u}$ is the vertical shear of alongfront velocity, $\mathbf{u}=(0, \bar{v}, 0)$. Here, overbars denote mean quantities. This is the classic criterion for symmetric instability of a front in TWB given in terms of nondimensional numbers (Hoskins 1974). It has also motivated a decomposition of the

\footnotetext{
${ }^{1}$ In Eq. (1), the bracketed quantity can be rewritten in terms of the radial gradient of the square of absolute angular momentum, thereby making a direct connection between Eq. (1) and Rayleigh's original statement that the fluid "is stable only under the condition that the circulation always increases with $r$ " (Rayleigh 1917; Chandrasekhar 1961). Here, Rayleigh (1917) uses the term circulation to refer to the angular momentum.

${ }^{2}$ The Ooyama-Hoskins model is even more specific. It is characterized by constant vertical and horizontal gradients in velocity and density.
} 
occurrences of negative PV into separate categories that define which terms are most important in reducing PV (Thomas et al. 2013), including gravitational, inertial, and symmetric instabilities. The reader is also referred to $\mathrm{Li}$ et al. (2012) and Hamlington et al. (2014) for presentations of these instability types in the presence of surface wave effects.

Somewhat coincident with these studies, Yanai (1961) appears to have addressed a more challenging problem. In seeking to understand formation mechanisms of tropical cyclones, Yanai derived an Ooyama-Hoskins-like equation valid for fronts with curvature. From this, he obtained an instability criterion valid for fronts in cyclogeostrophic (hereafter gradient wind balance; GWB) under certain restricted conditions. ${ }^{3}$ For a vortex, owing to the boundary imposed at $r=0$, it demands solutions in the form of Bessel functions and poses a unique mathematical problem. This is likely why Ooyama (1966), years later, concluded that an eigen- or normal-mode solution may not exist and proceeded to solve the sufficiency problem in a creative manner. This difficulty in solving the governing equation using classical normal-mode analysis was recently reiterated by Kloosterziel (2010, p. 173, paragraph 2). It thus remains an outstanding problem.

\section{c. Motivations for the present study}

Why then revisit this problem? If the criterion of Solberg (1936) [cf. Eq. (1)], proved sufficient by Fjortoft (1950), and Ooyama (1966), addresses the problem of symmetric instability, why reconsider the impact of curvature on frontal stability? While this criterion has been widely known in atmospheric circles for nearly a century, the role of curvature on dynamics at ocean fronts is typically neglected. Here, we do not have in mind classical baroclinic instability in which potential energy fuels the growing disturbance. This was addressed more recently by Grooms (2015). Rather, we have in mind baroclinic instabilities in which kinetic energy from the vertical shear in the flow supplies the necessary energy (Taylor and Ferrari 2010; Thomas and Taylor 2010; D'Asaro et al. 2011; Thomas et al. 2013; Bachman et al. 2017; Buckingham et al. 2019). Notable exceptions to this statement include studies by Holmes et al. (2014), Shakespeare (2016), Adams et al. (2017), and Brannigan et al. (2017), which we briefly describe below.

Holmes et al. (2014) investigated changes in PV near tropical instability waves (TIW) within high-resolution regional simulations, finding that the centripetal acceleration modified the results in locations confined to sharp baroclinic fronts. Shakespeare (2016) examined the cyclogeostrophic adjustment and frontogenesis problem for curved density fronts, essentially generalizing the work of Hoskins and Bretherton (1972). Adams et al. (2017) examined submesoscale instabilities on the edge of a mesoscale eddy in the Southern Ocean. Although Adams et al. (2017) did not examine the relevant criterion given above, the

\footnotetext{
${ }^{3}$ Yanai (1961) makes several approximations in his derivation that restrict his solution. For example, Yanai (1961, his section 1.2.4) assumes vertical and lateral shears and stratifications to be locally constant, which may be inappropriate since GWB cannot generally be satisfied in this manner.
}

authors did examine the Ertel PV, finding that curvature did not appreciably modify their results. Last, Brannigan et al. (2017) explored these dynamics within numerical simulations of mesoscale eddies, deriving a useful expression for the growth rate of symmetric disturbances under limiting conditions relevant to mesoscale eddies. In summary, although several studies have considered the effect of curvature on frontal stability, the vast majority of oceanographic studies employ TWB to understand symmetric instability, something that may be a poor approximation for small-scale eddies or sharply curved fronts. Thus, the first motivation is to reexamine frontal stability and instability in the oceanic context and that may lead to new discoveries. We find, for example, that it may help to explain why submesoscale coherent vortices tend to be anticyclonic, thereby potentially solving a puzzle about the distribution of relative vorticity at small horizontal scales in the ocean (McWilliams 1985, 2016).

A second motivation for this study is slightly academic but nonetheless relevant. Numerous studies have employed the statement " $f q<0$ implies potential for instability" without precisely understanding the 1 ) origin or 2) range of validity of this statement. With regard to origin, admittedly, the study of Hoskins (1974) provides a clear case in which this statement is true, serving as a useful reference. We ourselves have employed this technique in earlier studies. However, such arguments are restricted to the specific case of a front in TWB. It can, however, be shown that the stability of a front can be deduced in part from Ertel's PV theorem applied to a thermodynamic variable (e.g., density) - so long as the fluid is inviscid and baroclinic fluid. That is, in the absence of friction and for a fluid where isopycnals are inclined to pressure surfaces (i.e., $\nabla \rho \times \nabla p \neq 0$ ), PV conservation demands density conservation and instabilities are not possible. In contrast, where $q$ is not conserved, density is no longer conserved, implying that turbulent mixing (diabatic) processes are possible.

However, this statement does not address the sufficiency question. We find, for example, that the range of validity of the $P V$ criterion is limited. In particular, the criterion given by Hoskins (1974) is not sufficient for curved baroclinic flow because it neglects centrifugal forces that fluid parcels experience within such fronts. In summary, for curved fronts, $f q<0$ is not the correct criterion. One must account for centrifugal effects.

A third and final motivation is application driven. Frontal instabilities such as those described in this study are ubiquitous in the World Ocean and may have implications for climate. In recent years, submesoscale instabilities have been identified as having impacts on upper-ocean stratification (Fox-Kemper et al. 2011), biogeochemistry and tracer exchange (Klein and Lapeyre 2009; Lévy et al. 2012; Mahadevan 2016; Smith et al. 2016), and energetics associated with the wind-driven ocean circulation (Thomas and Taylor 2010). Given that these specific areas each play a role in shaping Earth's climate, it is conceivable that their integrated effect may be important. Thus, a longer-term but nonetheless relevant goal of this study is to better understand the role submesoscale processes might play in this climate system.

The historical introduction given above is largely concerned with the stability of cylindrical vortices. However, GWB balance also has applicability to meandering fronts since, as in the 


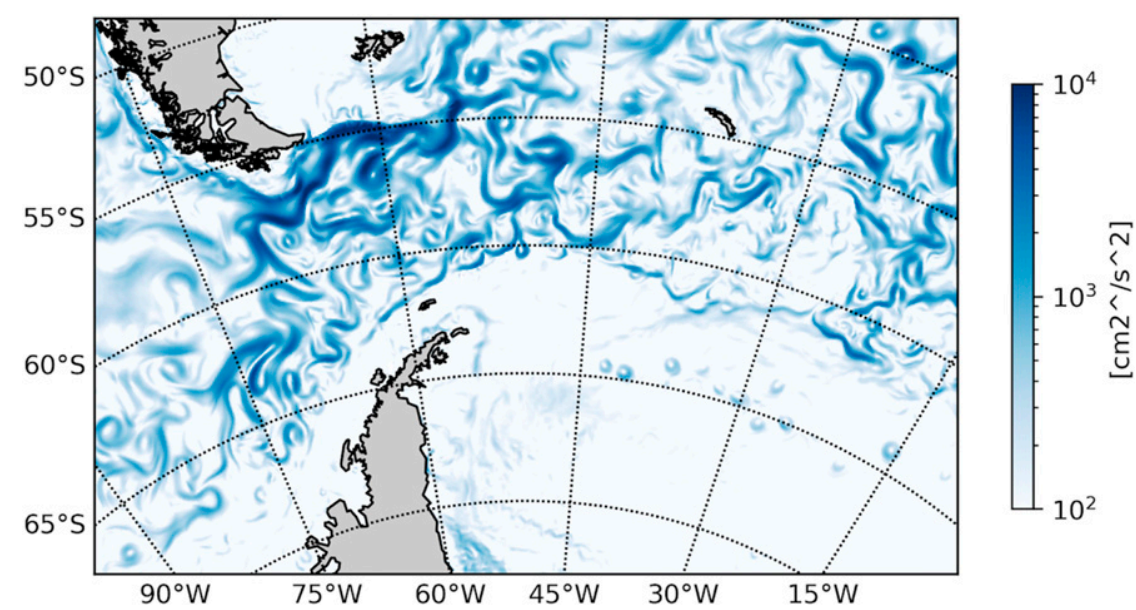

FIG. 1. Surface kinetic energy within a realistic, regional model of the Southern Ocean. Surface kinetic energy has been estimated as $\mathrm{KE}_{s}=\left(u_{s}^{2}+v_{s}^{2}\right) / 2$, where $u_{s}$ and $v_{s}$ are daily averages of zonal and meridional velocities following 1.25-yr spinup. The model is known as the Coastal and Regional Ocean Community Model (CROCO), the French branch of the Regional Ocean Modeling System (ROMS). The simulation was initialized and forced at lateral boundaries using the Southern Ocean State Estimate (SOSE) (Verdy and Mazloff 2017), forced at the ocean surface using atmospheric reanalysis from the European Centre for Medium-Range Weather Forecasts (Dee et al. 2011), and sea surface salinity has been nudged toward a climatological state. The graphic is used here simply to illustrate curved fronts and vortices that are typically encountered in the ACC.

atmosphere, GWB is a useful approximation for the momentum balance in the crests and troughs of ocean fronts. Such flows can be found in boundary currents such as the Gulf Stream Current and Kuroshio, or in the strong zonal flows of the Antarctic Circumpolar Current (ACC). A quick glance at surface kinetic energy in the ACC (Fig. 1) (see also Dong et al. 2006; Sokolov and Rintoul 2009; Cornillon et al. 2019, their section 3.5), for example, will illustrate to the reader that GWB is likely a reasonable model. In such cases, the radius of a vortex $r$ should be replaced by a radius of curvature $R$ and the azimuthal velocity should be replaced by an alongfront velocity $\bar{v}$. This is illustrated schematically in Fig. 2 and described in greater detail in appendix B. TWB can then be recovered in the limit of $R \rightarrow \infty$. We acknowledge that a more complete description of the role of curvature demands use of the natural coordinates (Holton 1992; Wenegrat and Thomas 2017). However, for simplicity, we restrict our presentation of the problem to one in cylindrical coordinates.

\section{d. Outline of the study}

Our study is broken up into two parts because of length. In the first part of the study (this paper), we review theoretical concepts and present a nondimensional form of an instability criterion valid for curved fronts. These largely reflect the authors' efforts to understand the sufficient criterion in light of the acknowledged importance of PV to symmetric instability (Hoskins 1974, 2015). In a sense, the paper approaches the problem from a "frontal dynamics" viewpoint rather than through angular momentum conservation (Rayleigh 1917). In the second part of the study (Buckingham et al. 2021, hereinafter Part II), we investigate interesting outcomes of the criterion applied to idealized fronts and vortices in GWB. In particular, we examine stability in the low-Richardson-number regime characteristic of symmetric instability.

The outline of the present portion of the study is as follows. In section 2, we consider the statement that the Coriolis parameter multiplied by the Ertel PV is positive for stable flow but that negative values of this product indicate potential for instability. We first do so in the context of Ertel's PV theorem (section $2 \mathrm{a}$ ), articulating that density conservation and PV conservation are inseparable for inviscid, baroclinic flows, such that there can be no turbulent diabatic process so long as $q$ is conserved. In section $2 \mathrm{~b}$, we examine governing equations for the mean circulation within a front in TWB. Referencing work by Hoskins and Bretherton (1972), we show that the elliptic and hyperbolic nature of the governing equation contains information closely tied to frontal stability and instability (Holton 1992). Alternatively, this can be viewed as an ability to invert the partial differential equation (PDE) for positive values of $f q$ but not for negative values of $f q$ (Hoskins et al. 1985; Thorpe and Rotunno 1989). In section 2c, we apply these same arguments to a front in GWB. Starting from the inviscid, nonhydrostatic, Boussinesq equations, we derive a governing equation for the cross-frontal overturning or secondary circulation within the front (appendix A). We then obtain a hyperbolic criterion, which we note is a generalized form of the Rayleigh criterion first given by Solberg (1936). For a limiting case applicable to symmetric instability, we also demonstrate that this is a sufficient criterion. In words, this criterion states that, for axisymmetric vortices or curved fronts with symmetry in the alongfront direction, both the Ertel PV and absolute angular momentum play governing roles in the stability of the front. 


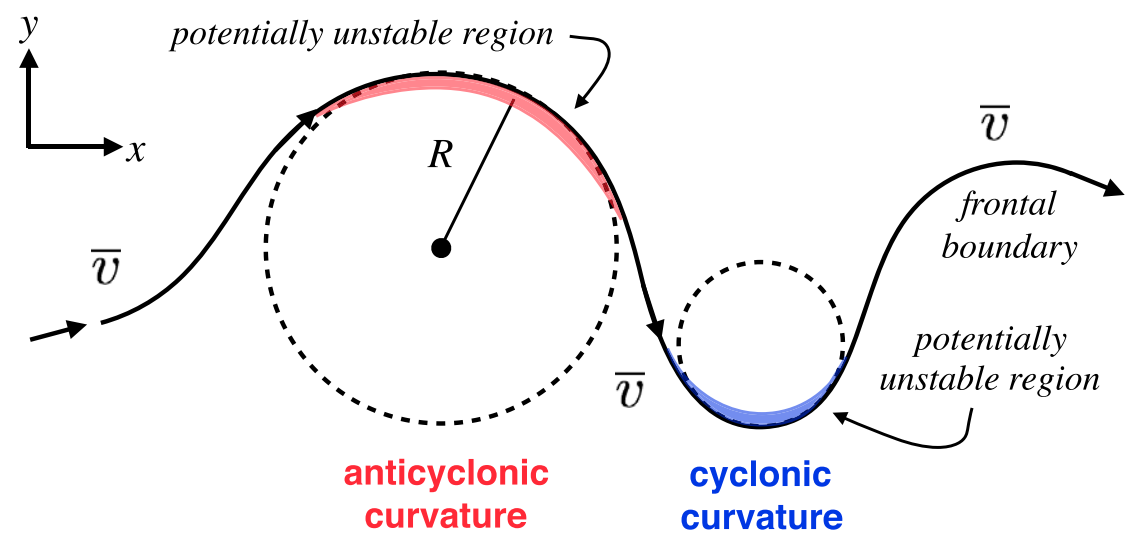

FIG. 2. Illustration of mean flow $\bar{v}$ and radius of curvature $R$ of a meandering front in the Northern Hemisphere $(f>0)$, as well as potential instabilities at locations within this front. The first meander has anticyclonic curvature $(R<0)$, and the second meander has cyclonic curvature $(R>0)$. In this graphic, $x$ and $y$ correspond to alongfront and cross-front displacements, consistent with Eq. (B7).

If either of these is negative (but not both), we have the potential for instability. Moreover, since the criterion, $\Phi<0$, is sufficient (Fjortoft 1950; Ooyama 1966; see also Cho et al. 1993; Kloosterziel 2010), we can confidently state that instability is guaranteed for fronts with symmetry in the direction of the gradient wind shear. This applies to fronts and vortices on the $f$ plane. This subsequently forms the basis for a nondimensional criterion presented in section 3 and discussed at length in section 4. Section 5 summarizes and concludes the study, while appendix B suggests a modification to an existing instability classification method (Thomas et al. 2013) that is consistent with this criterion.

\section{Theory}

The classical method of deriving an instability criterion involves the following recipe: 1) begin with the governing equations (momentum, energy, and continuity), 2) decompose variables into basic (mean) state and perturbations from these mean states, 3) eliminate products of perturbations, 4) combine these equations in a way that results in a single governing equation, and 5) demonstrate that a growing solution to this equation exists only under certain circumstances. This is the classical linear, normal-mode instability approach and, relevant to the present study, has been used by Ooyama (1966) and Hoskins (1974) for a front in TWB. Moreover, this approach leads to additional insight about the energetics of the flow (Li et al. 2012; Thomas et al. 2013). While we ourselves have made an attempt at this, completing steps 1-4 in appendix A, and step 5 for a limiting case, such an approach has proven difficult for the general problem owing to boundary conditions, making solutions inseparable in $r$ and $z$ (Ooyama 1966; Kloosterziel 2010; Nelson et al. 2013). Progress can be made by locally approximating vertical and radial gradients in buoyancy and velocity as constant (e.g., Brannigan et al. 2017), but such approximations necessarily restrict the solution. Thus, we have instead adopted the following approach.
We first develop arguments that the discriminant of the governing equation for the mean circulation within a front in TWB contains information about the stability of the flow. We then apply this same logic to a front in GWB, first deriving the governing equation, then obtaining the discriminant. Since the hyperbolic condition corresponds to the generalization of the Rayleigh criterion first proposed by Solberg (1936) and later proved sufficient by Fjortoft (1950) and Ooyama (1966), we then have a sufficient criterion for instability valid for curved fronts in GWB. To place Part II of this work on terra firma, we additionally describe a normal-mode solution comparable to Hoskins (1974) that is valid for the case of symmetric instability (section e of appendix A). Prior to these steps, however, we first formally define the Ertel PV, demonstrating that this quantity is important for all baroclinic fronts.

\section{a. Ertel's PV theorem}

Both rotation and stratification play important roles in the stability of fluid parcels. If one of these environmental characteristics reduces to zero, fluid parcels are no longer bound but become unstable, translating away from their balanced or equilibrium positions. These concepts of rotational and static stability are built into the definition of Ertel PV.

Starting with the Navier-Stokes equations, it is possible to derive a conservation equation for the absolute vorticity:

$$
\frac{D \boldsymbol{\omega}_{a}}{D t}=\boldsymbol{\omega}_{a} \cdot \nabla \mathbf{u}-\boldsymbol{\omega}_{a} \nabla \cdot \mathbf{u}+\frac{\nabla \rho \times \nabla p}{\rho^{2}}+\nabla \times \frac{\mathscr{F}}{\rho},
$$

where $\boldsymbol{\omega}_{a}=2 \boldsymbol{\Omega}+\nabla \times \mathbf{u}$ is the absolute vorticity, $\rho$ is density, $p$ is pressure, and $\mathscr{F}$ is the frictional force acting on a fluid parcel (Pedlosky 1987; Müller 1995; Kundu and Cohen 2008). Assuming frictional forces are zero $(\mathscr{F}=0)$ and using the continuity equation, one can rearrange this to obtain the conservation of absolute vorticity per unit mass of an inviscid fluid:

$$
\frac{D}{D t}\left(\frac{\boldsymbol{\omega}_{a}}{\rho}\right)=\left(\frac{\boldsymbol{\omega}_{a}}{\rho} \cdot \nabla\right) \mathbf{u}+\frac{\nabla \rho \times \nabla p}{\rho^{3}} .
$$


Defining a scalar quantity $\lambda$ as $(D \lambda / D t)=\Psi$, one can manipulate the equations [see, for example, Pedlosky (1987) or Müller (1995)] to obtain

$$
\frac{D \Pi}{D t}=\frac{\boldsymbol{\omega}_{a}}{\rho} \cdot \nabla \Psi+\nabla \lambda \cdot \frac{\nabla \rho \times \nabla p}{\rho^{3}},
$$

where $\Pi$ is the generalized Ertel $P V$ :

$$
\Pi=\frac{\boldsymbol{\omega}_{a}}{\rho} \cdot \nabla \lambda
$$

Equation (6) states that the quantity $\Pi$ is conserved so long as the right-hand side is zero. This occurs when 1 ) frictional forces are absent, 2) $\lambda$ is a conserved quantity (such that $(D \lambda / D t)=$ $\Psi=0$ ), and either 3a) the fluid is barotropic (i.e., $\nabla \rho \times \nabla p=0$ ) or $3 b$ ) the fluid is baroclinic (i.e., $\nabla \rho \times \nabla p \neq 0$ ) but $\lambda$ is a function of pressure and density (i.e., a "thermodynamic variable" so that $\nabla \lambda$ is always perpendicular to $\nabla \rho \times \nabla p)$. In the present study, we are concerned with the baroclinic case, so that specifying $\lambda$ in terms of density ${ }^{4}$ or buoyancy (e.g., $\lambda=\rho g$ ) leads to conservation of PV [cf. Eq. (2)]:

$$
\frac{D \Pi}{D t}=\frac{D}{D t}\left(\frac{\boldsymbol{\omega}_{a}}{\rho} \cdot \nabla \lambda\right) \rightarrow \frac{D q}{D t} .
$$

The point we wish to make here is that, for a baroclinic fluid in which density surfaces are inclined to pressure surfaces, conservation of buoyancy or density by definition implies conservation of PV, and vice versa: $D q / D t=0 \leftrightarrow D b / D t=0$. That is, conservation of PV necessitates adiabatic flow and mixing of density is not possible. Assuming $q$ in the Northern Hemisphere to be initially positive, and $q$ in the Southern Hemisphere to be initially negative, then $f q$ must be positive for any future state. Thus, PV conservation places a strong constraint on the sign of $f q$, though, by itself, does not yield a sufficient instability criterion.

Away from boundaries in the ocean and atmosphere, the Ertel PV is approximately conserved following a fluid parcel. Moreover, at large horizontal scales, it has the same sign as Earth's vorticity in the respective hemispheres-that is, PV is positive in the Northern Hemisphere and negative in the Southern Hemisphere. However, in the presence of friction and buoyancy loss, the product $f q$ can be modified from its stable state, reduced to zero, and even become negative. It is at this point that one expects loss of balance and potential for instability. Because of its dynamical importance for ocean circulation and water-mass transformation, a framework exists to quantify how $q$ is modified in the oceans, and we refer the reader to these excellent studies for further information

\footnotetext{
${ }^{4}$ The coefficient of thermal expansion for seawater increases with pressure (McDougall 1987) such that it introduces an additional term in Eq. (8) (Marshall and Nurser 1992; Straub 1999). This is relevant since oceanic fronts and vortices can be found within the deep and intermediate oceans. Where possible, we therefore recommend neutral density $\gamma$ be used, where $\rho$ in Eq. (8) should be replaced by $\gamma$ (or similar) (Jackett and McDougall 1997; Eden and Willebrand 1999).
}

(e.g., Haynes and McIntyre 1987; Marshall and Nurser 1992; Deremble et al. 2014; Morel et al. 2019).

\section{b. Ellipticity and hyperbolicity of the governing equation: Thermal wind balance}

The potential for instability can be inferred from the nature of the equations governing balanced flow. The equation moves from an elliptic to hyperbolic form and it is at this point that one anticipates instability. Although such methods are restrictive - they cannot be used to establish sufficiency - they nevertheless provide insight into the dynamics.

A model of frontogenesis was extensively studied by Hoskins and Bretherton (1972). While a number of concepts are examined in their study, one subject they considered was the crossfrontal circulation or overturning within a front as a result of frontal intensification. The dynamics governing overturning or cross-frontal circulation within a front in TWB can be expressed in terms of a streamfunction $\psi$ [cf. Hoskins and Bretherton 1972, their Eq. (3.45)]:

$$
\partial_{\chi}\left(\frac{q}{f} \partial_{\chi} \psi\right)+\partial_{Z}\left(\frac{f^{2} \theta_{o}}{g} \frac{1}{r} \partial_{z} \psi\right)=-2 \alpha \partial_{\chi} \theta
$$

Here, $\chi=x+\bar{v} / f$ denotes a generalized horizontal coordinate, $x$ is the cross-frontal distance, $\bar{v}$ is the alongfront velocity, $q$ is the Ertel PV, $\theta$ is potential temperature, $\theta_{o}$ is a reference temperature (assumed to be constant), $g$ is gravity, $r$ is pseudodensity, $z$ is the vertical coordinate, and $\alpha$ represents forcing by the mesoscale eddy field. This equation is a steady-state version of the linearized governing equation typically examined in studies of symmetric instability (Ooyama 1966; Hoskins 1974; Mooers 1975; Thomas et al. 2013; Li et al. 2019) and is also a form of Sawyer-Eliassen equation (Sawyer 1956; Eliassen 1962).

Equation (9) is a second-order PDE that can be described as elliptic, parabolic, and hyperbolic, with corresponding implications for the type of solutions expected for $\psi$ (e.g., Kreyszig 1998). Balanced flow is expected when the PDE is elliptic, linear waves are anticipated when the PDE is parabolic, and growing or decaying wave-like solutions are expected when the PDE is hyperbolic. The latter case, therefore, represents potential for instability. It can readily be shown that the $\mathrm{PDE}$ is hyperbolic when $f q<0$, where one makes the substitution $\theta_{o} \rightarrow \rho_{o}$ and $r \rightarrow \rho$. The condition $q=0$ yields the marginally stable state corresponding to linear internal waves (Müller 1995).

A physical interpretation of this singularity is as follows. In this expression, the balanced flow is assumed to be forced by the larger-scale flow field [i.e., the right-hand side of Eq. (9)]. In an attempt to conserve PV, the horizontal and vertical shears within the front become pronounced, so much so that the front eventually becomes unstable. The associated frontal stratification and shears are quantified by $\mathrm{Ro}$ and $\mathrm{Ri}$, and the relation between the two quantities is given by the nondimensional PV: $q^{\prime}=1+\mathrm{Ro}-\mathrm{Ri}^{-1}$ [cf. Eq. (3)]. As Ri decreases in magnitude, Ro must correspondingly increase in magnitude in order to conserve $q^{\prime}$, and does so in an unbounded fashion, leading to skewness in the distribution of relative vorticity. Note that Ro can only increase unbounded in the positive direction 
because $\mathrm{Ri}>0$. This interplay between Ro and Ri in the presence of PV conservation is summarized nicely by Thomas et al. (2008, their Fig. 4e). Note that this is exactly what is stated by Hoskins and Bretherton (1972, p. 19, item 11), where there they have in mind Kelvin-Helmholtz instability. ${ }^{5}$ In summary, the PDE contains dynamical information about mean shears and stratification within the front, and this information is contained within the discriminant.

\section{c. Ellipticity and hyperbolicity of the governing equation: Gradient wind balance}

In an effort to generalize results of Hoskins and Bretherton (1972) to curved fronts, Shakespeare (2016) derived a nonlinear governing equation for the mean flow of a front in GWB. While the governing PDE is not cast in terms of the overturning circulation, $\psi$, it does describe the mean azimuthal flow and does so in terms of the Ertel PV. It is therefore analogous to Eq. (9) but is valid for flow in GWB. As noted by Shakespeare (2016), the PDE is elliptic if $q / f>0$, hyperbolic if $q / f<0$, and parabolic for $q=0$. Since the requirement $q / f<0$ can be rewritten as $f q<0$, one finds that the hyperbolic constraint on the PDE is, again, $f q<0$. While the above description is true, an additional constraint is implicit within the work of Shakespeare (2016) that is not readily apparent from our discussion. His transformed coordinate system assumes conservation of absolute angular momentum, $L$, so that the hyperbolic constraint is $f q<0$ subject to the condition $f L>0$. We briefly discuss this additional constraint below.

\section{COMBINED IMPORTANCE OF ABSOLUTE ANGULAR MOMENTUM AND ERTEL PV}

There are added constraints that arise in special circumstances and these can modify the stability criterion. For studies in which azimuthal symmetry is present, the absolute angular momentum, $L=r \bar{v}+f r^{2} / 2$, must also be conserved (Holton 1992). This can be shown by rewriting the azimuthal momentum equation with $\partial_{\theta} \rightarrow 0$ as $D L / D t=0$ (Rayleigh 1917). To aid in the present study, we have derived a governing equation similar to Eq. (9) but applicable to an inviscid, axisymmetric vortex in GWB and absent forcing (appendix A). Although comparable derivations can be found elsewhere (Eliassen 1951; Yanai 1961; Shapiro and Willoughby 1982), it is notable that our derivation permits a nonhydrostatic pressure perturbation.

Since the governing equation is somewhat lengthy [cf. Eq. (A15)], we summarize the final result. Examining the discriminant of the governing equation, and using arguments of ellipticity and hyperbolicity developed above, we obtain the following hyperbolic condition:

$$
\Phi=[(f+2 \bar{v} / r)(f+\bar{\zeta})]_{\rho}=\frac{2 L q}{r^{2}}<0
$$

\footnotetext{
${ }^{5}$ Although their statement might have been made prior to a complete understanding of symmetric instability and its relation to PV (Hoskins 1974), it is nonetheless complementary since symmetric instability is thought to result in Kelvin-Helmholtz instability, which is eventually arrested at the dissipative scale (Taylor and Ferrari 2009).
}

[cf. Eqs. (A18) and (A23)], or simply

$$
L q<0
$$

Also, see the cases discussed by Kloosterziel et al. (2007) and Kloosterziel (2010). Given that absolute angular momentum $L$ typically shares the same sign as $f$ (Holton 1992), this criterion states that both the absolute angular momentum and Ertel PV (or neither) must be conserved in the stable, axisymmetric baroclinic vortex on the f plane. Cross referencing this expression with Eq. (1), one notes that this is the generalization of the Rayleigh (1917) criterion first proposed by Solberg (1936). Moreover, we can see an obvious connection to the frontal dynamics discussed in the context of PV (Hoskins and Bretherton 1972; Hoskins 1974). Whereas shears and stratification were contained in the TWB discriminant, now shears, stratification, and centrifugal forces are accounted for in the GWB discriminant. When the discriminant is zero or becomes of opposite sign, fluid parcels are no longer bound to their balanced state but can translate radially from the vortex center.

It is noteworthy that not even Rayleigh's criterion for the instability of a barotropic flow was initially given as a sufficient criterion; his criterion was based on physical arguments (Rayleigh 1917; Chandrasekhar 1961). This is comparable to the above except that here we have outlined the governing equation that leads to this criterion, despite that a normal-mode solution may not be immediately accessible. Moreover, in a limiting case applicable to symmetric instability, we have established its sufficiency (section e of appendix A). In this regard, we have done our work since Part II is principally concerned with symmetric instability.

\section{d. Spinning-top analogy}

We find the following helpful when attempting to convey these dynamics to an audience that is not immersed in these concepts. Perhaps it will help readers, as well. A heuristic but nonetheless helpful way to understand the discussion above is by way of analogy to a spinning top. In general, the conservation of angular momentum applies and the top continues to rotate on a surface indefinitely. However, owing to friction, the top will soon slow down and will cease to spin in an absolute sense; it is then susceptible to disturbances in the form of atmospheric pressure perturbations or surface roughness that allow it to topple over. For fluids in the ocean and atmosphere, the dynamic is similar, only here the disturbances are internal waves and the conserved quantities are the Ertel PV and absolute angular momentum. As PV is the product of vorticity and buoyancy gradients [cf. Eq. (2)], if either rotation or stratification are zero-or their inner product-then fluid parcels are no longer bound to a balanced state but can translate away from their equilibrium position. For curved flows, we additionally have the constraint that absolute angular momentum of fluid parcels must be conserved. (In other words, the flow cannot "spin" faster than what its initial angular momentum allows). For stratified, rotating flow, this implies that if density surfaces are inclined to angular momentum surfaces, then fluid parcels can move outwardly along constant density surfaces, reduce their angular momentum, and result in instability. 
Although the associated instability criterion might have been obtained in other ways (Solberg 1936; Fjortoft 1950; Cho et al. 1993), here we have obtained the relevant expression from a hyperbolic condition on the governing PDE, helping build insight into the physics while at the same time making evident the assumptions and limitations in this expression. Finally, we have pointed out that, in a limiting case, a normal-mode solution exists and we recover the criterion, $\Phi<0$ (section e of appendix A). In particular, for this simplified model, the growth rates for disturbances aligned with isopycnals are found to scale with $-f^{2} \Phi^{\prime}$, where the primed notation denotes a nondimensional form of $\Phi$ expressed below. In summary, for curved fronts, both the Ertel PV and absolute angular momentum must be conserved to ensure stability. This yields a generalization of the Rayleigh criterion, $\Phi<0$, first given by Solberg (1936) and later shown sufficient by Fjortoft (1950), Ooyama (1966), and Cho et al. (1993).

\section{Dimensional and nondimensional forms}

The arguments given above were presented in order to clarify the connection of Ertel PV, $q$, and absolute angular momentum, $L$, to the present problem. Under these assumptions, we now express full dimensional and nondimensional forms of the instability criterion for a flow in GWB. While we have previously given a dimensional form of the criterion [e.g., Eq. (A17)], we do so again, demonstrating that one can start directly from Eq. (10).

We first define a coordinate system $\mathbf{x}=(r, \theta, z)$, such that the unit vectors in these directions $(\hat{\mathbf{r}}, \hat{\boldsymbol{\theta}}, \hat{\mathbf{z}})$ form an orthonormal basis. We also define the velocities in these directions as $\mathbf{u}=(u, v, w)$. The mean Ertel PV for a flow in GWB is given by

$$
q=(f+\bar{\zeta}) N^{2}-\left(f+\frac{2 \bar{v}}{r}\right)\left|\partial_{z} \bar{v}\right|^{2}
$$

where, again, overbars denote mean quantities. As in appendix A, we have neglected the meridional component of Coriolis and have defined the lateral buoyancy gradient from the vertical derivative of the horizontal momentum equations:

$$
\partial_{r} \bar{b}=\left(f+\frac{2 \bar{v}}{r}\right) \partial_{z} \bar{v}
$$

This defines the mean state (i.e., GWB), where perturbations from this state are much smaller in magnitude than mean quantities. Confining our solution to stable stratification away from the equator, we can divide Eq. (12) by $f N^{2}$ to obtain the nondimensional Ertel PV for a flow in GWB:

$$
q^{\prime}=1+\mathrm{Ro}-(1+\mathrm{Cu}) \mathrm{Ri}^{-1}=0
$$

where we have defined Ro $=\bar{\zeta} / f$ as the gradient Rossby number, $\mathrm{Ri}=N^{2} /\left|\partial_{z} \bar{v}\right|^{2}$ as the gradient Richardson number, and $\mathrm{Cu}=2 \bar{v} /(f r)$ as the curvature number-that is, a signed, nondimensional parameter that quantifies the curvature of the flow. First, note that $\bar{v}$ is the mean alongfront component of the flow and includes both geostrophic and cyclostrophic components. Second, in the limit $\mathrm{Cu} \rightarrow 0$, Eq. (14) reduces to the Ertel PV under TWB. Third, $\mathrm{Cu}$ is not independent of Ro since Ro $=\bar{\zeta} / f=(f r)^{-1} \partial_{r}(r \bar{v})=\partial_{r} \bar{v} / f+\mathrm{Cu} / 2$. Nevertheless, it becomes useful for separating the effects of centrifugal accelerations and shear on the stability of the flow. We also note that an expression comparable to Eq. (14) is given by Lazar et al. [2013, their Eq. (C4)].

Both the Ertel PV and absolute angular momentum are conserved for curved stable fronts, but instability can result if one (but not both) of these quantities is negative. Beginning with Eq. (10), a necessary and sufficient criterion for instability is then

$$
\Phi=L^{\prime} f q=(1+\mathrm{Cu}) f q<0,
$$

where, again, $L=r \bar{v}+f r^{2} / 2$ is the absolute angular momentum (Holton 1992), $L^{\prime}=2 L /\left(f r^{2}\right)=1+\mathrm{Cu}$ is a nondimensional form of absolute angular momentum, and $\mathrm{Cu}$ is the curvature number defined above. Together with the expression for the PV under GWB [cf. Eq. (14)], we obtain an instability criterion that is valid for inviscid, baroclinic flow in GWB:

$$
\Phi=(1+\mathrm{Cu}) f q=\left(f+\frac{2 \bar{v}}{r}\right)(f+\bar{\zeta}) N^{2}-\left(f+\frac{2 \bar{v}}{r}\right)^{2}\left|\partial_{z} \bar{v}\right|^{2}<0 .
$$

Expressed in nondimensional form, we have

$\Phi^{\prime}=L^{\prime} q^{\prime}=\underbrace{(1+\mathrm{Cu})(1+\mathrm{Ro})}_{\text {barotropic component }}-\underbrace{(1+\mathrm{Cu})^{2} \mathrm{Ri}^{-1}}_{\text {baroclinic component }}<0$,

where we have expressed the discriminant $\Phi^{\prime}$ as a sum of barotropic and baroclinic components to help with discussions below. While one would be justified in referring to $\Phi$ as the "Solberg discriminant" or even the "generalized Hoskins discriminant" [Eq. (16) generalizes $f q<0$ ], we have chosen to adopt the phrase "generalized Rayleigh discriminant" when speaking of $\Phi$ and the associated criterion.

It is worth considering a few limiting cases of the criterion to check for consistency. Here, we refer to the dimensional form of the criterion [cf. Eq. (16)]. Away from fronts, $\left|\partial_{z} \bar{v}\right|^{2}=0$, and in the absence of vorticity the criterion reduces to $f^{2} N^{2}<0$. Away from the equator, this is simply the criterion for gravitational instability. Assuming stably stratified flow, $N^{2}>0$, and no vertical shear, the criterion reduces to the Rayleigh criterion for inertial instability of barotropic vortices: $\Phi / N^{2}=$ $(f+\bar{\zeta})(f+2 \bar{v} / r)<0$ (Kloosterziel and van Heijst 1991; Mutabazi et al. 1992). Last, in the TWB limit (i.e., $r \rightarrow \infty$ ), the expression simplifies to the criterion for symmetric instability (Hoskins 1974). While a full understanding of the generalized Rayleigh criterion requires a more thorough discussion, several comments relevant to Part II of our study are provided below.

\section{Comments on the nondimensional criterion}

Most geophysical flows are borne with $L$ having the same sign as $f$, except perhaps near the equator (Holton 1992). In this case, $L^{\prime}=2 L /\left(f r^{2}\right)=1+\mathrm{Cu}>0$. This can be rationalized by considering the absolute angular momentum of a fluid parcel on the sphere (http://glossary.ametsoc.org/wiki/Absolute_angular_momentum) 
and noting that it is largely determined by Earth's angular momentum. Now, for cyclonic flow $\mathrm{Cu}>0$, whereas for anticyclonic flow $\mathrm{Cu}<0$. Thus, examining the barotropic component of the stability discriminant, $(1+\mathrm{Cu})(1+\mathrm{Ro})$, one finds that $\mathrm{Cu}$ and Ro play similar roles in the stability of curved fronts, bounding the intensity of anticyclonic flow. One limits centripetal accelerations, while the other limits horizontal shear.

We next examine the baroclinic component of the stability discriminant $\Phi^{\prime}$, first starting with a discussion of the Ertel PV [cf. Eq. (14)]. Away from fronts, PV conservation requires $1+$ Ro $>0$. This simply reiterates what was said above. However, in the presence of lateral gradients in density, the baroclinic component of $q^{\prime}$ modifies this requirement, reducing the magnitude of Ro required for instability. One way to interpret this dynamically is that increased vertical shear and reduced vertical stratification act together to tilt the absolute vorticity vector away from the vertical, reducing the magnitude of Rossby numbers required for instability. Or conversely, reducing the range of Rossby numbers permitted for stability.

For a front in TWB, the baroclinic portion of PV tilts the vorticity vector away from the vertical. For a front in GWB, this tilting is now modified by the centripetal acceleration. Recall, $\mathrm{Cu}>0$ for cyclonic flow, whereas $\mathrm{Cu}<0$ for anticyclonic flow. Thus, relative to its thermal wind state, this tilting is away from the vertical (cyclonic) and toward the vertical (anticyclonic). Also, multiplication of the baroclinic component of PV by $L^{\prime}$ results in the term $-(1+\mathrm{Cu})^{2} \mathrm{Ri}^{-1}$. Since this is negative definite, it always reduces the stability of the front, but less so for anticyclones than for cyclones.

In summary, the role of curvature in modifying frontal stability is to twofold: it 1) limits the permitted range of Rossby numbers (barotropic) and 2) modifies this range through vortex tilting (baroclinic). The latter is the central focus of Part II.

\section{Itemized notes on the nondimensional criterion}

For reference, several aspects of the nondimensional Rayleigh criterion are summarized below:

1) Curvature number $\mathrm{Cu}$ is a signed quantity and typically shares the same sign as Ro. ${ }^{6}$ This is independent of hemisphere since it is divided by $f$. Therefore, one can unambiguously describe curvature as cyclonic $(\mathrm{Cu}>0)$ or anticyclonic $(\mathrm{Cu}<0)$.

2) For small $\mathrm{Cu}$ (i.e., as $r \rightarrow \infty$ ), we recover the classical criterion for symmetric instability valid under TWB (Hoskins 1974) [cf. Eq. (3)]. Equation (17) therefore supersedes the nondimensional form of Hoskins's criterion [cf. Eq. (3)].

\footnotetext{
${ }^{6}$ Coherent eddies or vortices are often observed with rings of negative relative vorticity immediately outside the radius of maximum velocity. In these regions, $\mathrm{Cu}$ and $\mathrm{Ro}$ can have different signs. While we suspect that the momentum balance within this region may depart from GWB, leading more to a "turbulent gradient wind" relationship, we nevertheless note that a reversal in vorticity ("shield") is present in many geophysical vortices.
}

Note, that it does so without assuming gradients in density and velocity are constant.

3) With regard to the barotropic portion of $\Phi^{\prime}$, curvature limits the intensity of anticyclonic flow, restricting the magnitude of Rossby numbers. Scaling the curvature number as $\mathrm{Cu} \sim$ $2 v_{m} /\left(f r_{m}\right)$, where $v_{m}$ and $r_{m}$ are characteristic scales for the azimuthal velocity and radius, one finds that bulk Rossby numbers scale as $\mathrm{Ro}_{b} \sim v_{m} /\left(f r_{m}\right)>-0.5$.

4) With regard to the baroclinic portion of $\Phi^{\prime}$, curvature is important when the gradient Richardson number $\mathrm{Ri}$ is low. Since the sign of $\mathrm{Cu}$ differs for cyclones and anticyclones, the possibility of two interesting phenomena emerge:

(i) anticyclonic flows can increase in nondimensional PV, and

(ii) cyclonic flows can decrease in nondimensional PV, potentially having $q^{\prime}<0$ for cases in which Ro $<$ $\mathrm{CuRi}^{-1}$. Given $\mathrm{Ri}$ near unity, this is clearly possible when $\mathrm{Ro}<\mathrm{Cu}$.

5) With regard to the full discriminant $\Phi^{\prime}$, The above effects in $\mathrm{i}$ and ii are slightly obscured in the full discriminant since $\mathrm{Cu}$ and Ro no longer cancel each other in the expression for $\Phi^{\prime}$. We instead observe that the two terms compete: $(1+\mathrm{Cu})(1+$ $\mathrm{Ro})$ and $-(1+\mathrm{Cu})^{2} \mathrm{Ri}^{-1}$, with the latter being negative definite, reducing the stability of the flow. For example, for cases in which $\mathrm{Cu}>\mathrm{Ro}$ and $\mathrm{Ri}=1$, we can expect instability. Note, however, that the magnitude of the baroclinic component of $\Phi^{\prime}$ remains sensitive to whether the flow is cyclonic or anticyclonic. That is, symmetric instability (elevated importance of baroclinic component) is enhanced for cyclonic flow when compared with anticyclonic flow.

Having established the criterion for instability applicable to curved density fronts, we now make two brief comments. First, it would be useful to modify the instability categories of Thomas et al. (2013) to account for curvature. While this is largely straightforward, a difficulty arises from the fact that, except in the limiting case described in section e of appendix A, a normal-mode solution to the governing equation [cf. Eq. (A15)] does not yet exist (Ooyama 1966; Kloosterziel 2010), thereby obscuring an obvious relationship between the energy for the disturbances and nondimensional numbers. However, because their technique proves powerful for helping to understand the dynamics associated with gravitational, centrifugal and symmetric instabilities, we propose a simple modification to their classification method consistent with $\Phi^{\prime}<0$ (appendix B and Table B1).

The second comment pertains to the stability of intense anticyclones. As pointed out by a reviewer, Eq. (17) suggests that for large enough $\mathrm{Ri}$, one can encounter a case of an anticyclone characterized by $\mathrm{Ro}<-1$ (negative absolute vorticity) and $\mathrm{Cu}<-1$ (negative absolute angular momentum) and yet still be stable since $(1+\mathrm{Cu})(1+\mathrm{Ro})>(1+\mathrm{Cu})^{2} \mathrm{Ri}^{-1}$. Indeed, we see this effect in portions of our parameter space for Ro $<-1$ and $\mathrm{Ri}>2$, suggesting intense anticyclones can be stable so long as both the PV and absolute angular momentum are not conserved. (Such a phenomenon is evident in stability maps for certain vortices presented in Part II.) Thus, this is a unique but theoretically possible phenomenon. One can rationalize, however, that most geophysical flows will begin with 
$f q>0$ and $f L>0$, or equivalently $L^{\prime}>0$ and $q^{\prime}>0$, thus approaching the marginally stable state from the "other side" of the parameter space.

\section{Summary and conclusions}

In this study, we have examined the role of curvature in modifying frontal stability. We first reconsidered the statement that the Coriolis parameter, $f$, multiplied by the Ertel potential vorticity $(P V), q$, is positive for stable flow and that instability is possible when this product is negative. We revisited Ertel's PV theorem, noting that density and PV are intimately linked to the point that PV conservation implies density conservation and vice versa. In the absence of friction and buoyancy loss, and assuming the scalar $f q$ starts from a positive value, it must remain positive and turbulent diabatic processes (i.e., mixing) are not possible.

Quoting Hoskins, "the basic zonal flow considered. . .cannot be generated from this state of no [meridional or cross-frontal] motion by any frictionless, adiabatic motion what[so]ever" (Hoskins 1974, p. 481), where the text in brackets has been added for clarity. The logical extension of this argument is also summarized nicely by Hoskins (1974): "[F]rictional and heating effects are needed to generate instability to symmetric motions in a previously stable atmosphere." In summary, although insufficient to guarantee instability, one might rationalize a portion of the preceding statement directly from Ertel's PV theorem, assuming an initially positive state, $f q$.

Second, we examined the governing equation for the secondary circulation within a front in thermal wind balance (TWB). By recalling work by Hoskins and Bretherton (1972), we noted that the transition of the equation from elliptic to hyperbolic form corresponds to the marginally stable state $(q=0)$. As stated above, this can equivalently be thought of as an ability to invert the PDE for positive values of $f q$ but not for negative values of $f q$ (Hoskins et al. 1985; Thorpe and Rotunno 1989; Shakespeare 2016). This subsequently motivated us to derive a governing equation for the overturning circulation within a front in gradient wind balance (GWB). While the full details are presented in appendix A, the derivation is valid under the following limiting conditions: nonhydrostatic, Boussinesq, inviscid, and axisymmetric flow (i.e., having alongfront symmetry) on the $f$ plane. Next, in attempting to solve the linear instability problem, we discovered (i) this may not be possible for the general problem using normal-mode methods and (ii) the hyperbolic condition on this PDE is well known. It corresponds to the criterion first proposed by Solberg (1936), which is itself a generalization of (i) Rayleigh's criterion by accounting for baroclinicity and (ii) Hoskins's criterion by accounting for centrifugal effects. Since the criterion has been established as sufficient (Fjortoft 1950; Ooyama 1966; Cho et al. 1993), we can confidently state that the expression is a necessary and sufficient criterion for instability. In words, one states that the product of Ertel PV and absolute angular momentum must be positive for stability within an inviscid front but that if either of these quantities (but not both) are negative, instability will result. Section e in appendix A provides a limiting solution to the governing equation, and we recover this criterion.
Third, we presented dimensional and nondimensional forms of the criterion. A useful dimensional form is

$$
\Phi=(1+\mathrm{Cu}) f q<0
$$

[cf. Eq. (16)], where $\mathrm{Cu}=2 \bar{v} /(f r)$ is a signed, nondimensional number quantifying the curvature of the flow. This nondimensional number emerges naturally from expressions of stability-both in the expression for PV and in absolute angular momentum. ${ }^{7}$ The reason this "generalized Hoskins criterion" is useful is that in the limit of $\mathrm{Cu} \rightarrow 0$ the criterion reduces to that valid for fronts in TWB (Hoskins 1974). Alternatively, one can write $L q<0$, although it is important to note that dimensional units are different.

A particularly useful nondimensional form of the criterion is given by Eq. (17):

$$
\Phi^{\prime}=L^{\prime} q^{\prime}=(1+\mathrm{Cu})(1+\mathrm{Ro})-(1+\mathrm{Cu})^{2} \mathrm{Ri}^{-1}<0,
$$

where $q^{\prime}$ is the nondimensional Ertel PV (for a flow in GWB), $L^{\prime}$ is a nondimensional form of absolute angular momentum, Ro is the gradient Rossby number, $\mathrm{Ri}$ is the gradient Richardson number, and $\mathrm{Cu}$ is defined above. We highlighted several aspects of this criterion, a few of which are detailed below.

1) We found that the baroclinic term is modified from its TWB state by the curvature of the flow and provided an interpretation in terms of vortex tilting. This expression also motivated us to revisit the instability categories (e.g., Thomas et al. 2013), and we obtained revised barotropic and baroclinic instability angles, $\phi_{o}$ and $\phi_{1}$ (appendix B; Table B1). In the limit $\mathrm{Cu} \rightarrow 0$, these angles reduce to those of Thomas et al. (2013), $\phi_{\mathrm{Ro}}$ and $\phi_{\mathrm{Ri}}$, such that they can be used in place of their angles. Recall that the relevant question being answered here is, "What aspect of the flow is most responsible for reducing the stability discriminant $\Phi^{\prime}$ ?"

2) We noted that the barotropic component of the stability discriminant, $(1+\mathrm{Cu})(1+\mathrm{Ro})$, contains two limits on Rossby numbers for stable flow. While one is the result of conservation of barotropic potential vorticity (leading to the statement $1+$ Ro $>0$ ), a limit on Rossby numbers is also imposed through conservation of absolute angular momentum $L$ (leading to the statement $1+\mathrm{Cu}>0$ ). In effect, it limits centripetal accelerations within an anticyclonic curved front. This statement implies that centripetal accelerations within anticyclonic curved fronts can never be greater than the magnitude of the Coriolis acceleration divided by two. This, in turn, places strong constraints on bulk Rossby numbers. For example, scale analysis shows that $\mathrm{Ro}_{b}>-0.5$ is a good "rule of thumb."

3) At low Richardson numbers, the baroclinic component of $\Phi^{\prime}$ has elevated importance and is dependent upon the curvature of the flow. Since $\mathrm{Cu}>0$ for cyclonic flow, while $\mathrm{Cu}<0$ for anticyclonic flow, this implies that there may

\footnotetext{
${ }^{7}$ The latter is comparable to how the gradient Rossby number, Ro, emerges when speaking of the stability of barotropic flow in the presence of PV conservation or absolute vorticity conservation.
} 
exist values of $\mathrm{Ri}$ for which cyclonically curved flows are less stable than anticyclonically curved flows for the same gradient Rossby number. This is particularly interesting to the authors and becomes the central focus of Part II.

\section{Discussion}

While each of the preceding points is certainly theoretically stimulating-with the third opening up new directions for studies involving symmetric instability-another potentially interesting corollary of this work is as follows. It is typically assumed that, away from boundaries, the Ertel PV is the conserved quantity: $D q / D t=0$ [cf. Eq. (8)]. However, the mathematical and physical arguments above suggest a different conservation principle is at work within curved fronts on the $f$ plane. In the absence of friction and diabatic processes, it is $\Phi$ that is conserved, leading to the statement $D \Phi / D t=0$, or $D \Phi^{\prime} / D t=0$ in nondimensional space.

To understand the implications of this statement consider the following. Imagine a fluid parcel contained within a meandering front (Fig. 2). As the fluid parcel proceeds along its path and in the absence of frictional and buoyancy forcing at the ocean surface, it is typically assumed that vorticity, stratification, and vertical shear (or lateral buoyancy gradients) change in an effort to conserve PV (i.e., in a Lagrangian sense). However, we instead observe that vorticity, stratification, vertical shear, and curvature (or centripetal accelerations) must change to conserve $\Phi$. In nondimensional form, one would say that Ro, $\mathrm{Ri}$, and $\mathrm{Cu}$ are altered to keep $\Phi^{\prime}$ constant. Equivalent arguments can be made for a vortex that has formed via frictional or buoyancy forcing within a boundary layer (e.g., Spall 1995; Thomas 2008; Gula et al. 2019), but that translates away from this environment.

These arguments, of course, assume that meridional departures of the fluid parcel from its mean location are finite in order that the assumption of constant Coriolis parameter is valid. They also assume GWB remains a good description of the momentum balance as the fluid parcel proceeds along its path. We can expect viscous effects to relax the stability criterion to some extent, where we have borrowed from theory and laboratory experiments of centrifugal instability (Taylor 1923). Additional understanding might be obtained by expressing the governing equations in spherical coordinates. Nevertheless, confining ourselves to submesoscale fronts or zonally oriented fronts away from the equator, and considering that PV is typically assumed to be the conserved quantity in the oceans, this approach to analyzing the dynamics of fluid parcels is new.

Acknowledgments. This study was made possible by a MarieSkłodowska Curie Actions (MSCA) Individual Fellowship from the European Commission (Proposal 798319). We are particularly grateful to the editor and two sharp reviewers for their careful consideration of the paper. We also acknowledge George Nurser (NOCS) and Stephen Griffies (GFDL), each of whom made comments several years ago that proved formative for this study. We thank Paul Billant (CNRS) for kindly sharing his copy of Solberg's original manuscript.

\section{APPENDIX A}

\section{A Generalized Rayleigh-Hoskins Criterion and a Limiting Solution}

Below, we obtain a stability criterion valid for an inviscid, stratified, axisymmetric vortex or curved density fronts with alongfront symmetry. Though our solution cannot be shown to be sufficient, it nonetheless matches the criterion first proposed by Solberg (1936) and later proved sufficient by Fjortoft (1950) and Ooyama (1966). Other approaches to obtaining this criterion include those based on ring-displacement arguments (e.g., Solberg 1936; Kloosterziel and van Heijst 1991) together with an energy-Casimir method (Fjortoft 1950), and Lyapunov stability methods (Cho et al. 1993; Kloosterziel 2010). We first derive an equation that governs secondary circulation within an axisymmetric vortex. The mean state is assumed to be in cyclogeostrophic or gradient wind balance (GWB) and secondary circulation is assumed to occur within a meridional plane perpendicular to the mean flow-that is, in radial and vertical directions, only. Last, we neglect the meridional component of the Coriolis vector $\tilde{f}=2 \Omega \cos \theta$, arguing the flow to be either sufficiently away from the equator or sufficiently stratified such that $N / \tilde{f} \gg 1$ (Colin de Verdière 2012). Similar derivations can be found by Eliassen (1951), Yanai (1961), and Shapiro and Willoughby (1982), among others.

\section{a. Governing equations}

Let us first define a coordinate system $\mathbf{x}=(r, \theta, z)$ such that unit vectors in these directions $(\hat{\mathbf{r}}, \hat{\boldsymbol{\theta}}, \hat{\mathbf{z}})$ form an orthonormal basis. We then define velocities in these directions as $\mathbf{u}=(u, v, w)$ (Lamb 1932). The equations governing nonhydrostatic, Boussinesq flow on an $f$ plane are then

$$
\begin{aligned}
D_{t} u-\frac{v^{2}}{r}-f v+\frac{\partial_{r} p}{\rho_{o}} & =0, \\
D_{t} v+\frac{u v}{r}+f u+\frac{\partial_{\theta} p}{\rho_{o}} & =0, \\
D_{t} w-b+\frac{\partial_{z} p}{\rho_{o}} & =0, \\
D_{t} b & =0, \quad \text { and } \\
\frac{1}{r} \partial_{r}(r u)+\frac{1}{r} \partial_{\theta} v+\partial_{z} w & =0,
\end{aligned}
$$

where $(D / D t)=\partial_{t}+\mathbf{u} \cdot \nabla=\partial_{t}+u \partial_{r}+(v / r) \partial_{\theta}+w \partial_{z}$ is the material or substantial derivative, $p$ is pressure, $f$ is the Coriolis parameter, $b=-g \rho / \rho_{o}$ is buoyancy, $g$ is acceleration due to gravity, $\rho$ is density, and $\rho_{o}$ is a reference density. If we further assume azimuthal symmetry, $\partial_{\theta}=0$, partition variables into mean and perturbations quantities (e.g., buoyancy, velocity, pressure) and eliminate products of perturbations, we obtain the following linearized set of perturbation equations:

$$
\begin{aligned}
& \partial_{t} u^{\prime}-\left(f+\frac{2 \bar{v}}{r}\right) v^{\prime}+\partial_{r} P=0, \\
& \partial_{t} v^{\prime}+(f+\bar{\zeta}) u^{\prime}+w^{\prime} \partial_{z} \bar{v}=0,
\end{aligned}
$$




$$
\begin{aligned}
\partial_{t} w^{\prime}-b^{\prime}+\partial_{z} P & =0, \\
\partial_{t} b^{\prime}+u^{\prime} \partial_{r} \bar{b}+w^{\prime} \partial_{z} \bar{b} & =0, \quad \text { and } \\
\partial_{r} u^{\prime}+\frac{u^{\prime}}{r}+\partial_{z} w^{\prime} & =0,
\end{aligned}
$$

where the mean state is given by

$$
\partial_{r} \bar{b}=\left(f+\frac{2 \bar{v}}{r}\right) \partial_{z} \bar{v} .
$$

Here, overbars denote mean quantities, primes denote perturbations from these mean values, $P=p^{\prime} / \rho_{o}$ is the reduced pressure perturbation (Gill 1982), and $\bar{\zeta}=\partial_{r} \bar{v}+\bar{v} / r$ is the mean relative vorticity. The time derivative of vertical velocity perturbation in the $z$-momentum equation permits a nonhydrostatic pressure perturbation, making the derivation differ from both Eliassen (1951) and Yanai (1961) (see also Lazar et al. 2013). This set of equations (A6)-(A10) can also be compared with the geostrophic equivalent (e.g., Mooers 1975).

We can combine these equations [cf. Eqs. (A6)-(A9)] into a single vorticity equation governing the meridional velocities $u^{\prime}$ and $w^{\prime}$, as follows. Although other methods are possible, here we follow the method of Lazar et al. (2013). See, for example, Eq. (2.11) of their study. We take $\partial_{t}\left\{\partial_{z}\right.$ [Eq. (A6) $-\partial_{r}[$ Eq. (A8)] $\}$ and then use $\partial_{r}\left[\right.$ Eq. (A9)] and $\partial_{z}\left[\right.$ Eq. (A7)] to replace $\partial_{t r} b^{\prime}$ and $\partial_{t z} v^{\prime}$, respectively. Doing so gives

$$
\begin{gathered}
\partial_{t}^{2}\left(\partial_{z} u^{\prime}-\partial_{r} w^{\prime}\right)-\left(\partial_{r} u^{\prime}\right) G-u^{\prime}\left(\partial_{r} G\right)-\left(\partial_{r} w^{\prime}\right) H-w^{\prime}\left(\partial_{z} G\right) \\
+\frac{2}{r}\left(\partial_{z} \bar{v}\right)\left[u^{\prime}(f+\bar{\zeta})+w^{\prime}\left(\partial_{z} \bar{v}\right)\right]+\left(f+\frac{2 \bar{v}}{r}\right)\left[\partial_{z} u^{\prime}(f+\bar{\zeta})\right. \\
\left.+u^{\prime}\left(\partial_{z} \bar{\zeta}\right)+\partial_{z} w^{\prime}\left(\partial_{z} \bar{v}\right)+w^{\prime}\left(\partial_{z}^{2} \bar{v}\right)\right]=0
\end{gathered}
$$

where we have defined $G=\partial_{r} \bar{b}$ and $H=\partial_{z} \bar{b}$ for brevity. The continuity equation [cf. Eq. (A10)] allows us to define a perturbation streamfunction $\psi$ such that

$$
\begin{aligned}
u^{\prime} & =\partial_{z} \psi \quad \text { and } \\
w^{\prime} & =-\frac{1}{r} \partial_{r}(r \psi)=-\left(\partial_{r} \psi+\frac{\psi}{r}\right) .
\end{aligned}
$$

Then, the governing equation reduces to a second-order PDE in $\psi$ :

$$
\begin{aligned}
& \partial_{t}^{2}\left(\partial_{z}^{2} \psi+\partial_{r}^{2} \psi+\frac{\partial_{r} \psi}{r}-\frac{\psi}{r^{2}}\right)-\left(\partial_{r z}^{2} \psi\right) G-\left(\partial_{z} \psi\right)\left(\partial_{r} G\right)+\left(\partial_{r}^{2} \psi+\frac{\partial_{r} \psi}{r}-\frac{\psi}{r^{2}}\right) H+\left(\partial_{r} \psi+\frac{\psi}{r}\right)\left(\partial_{z} G\right)+\frac{2}{r}\left(\partial_{z} \bar{v}\right)\left[\left(\partial_{z} \psi\right)(f+\bar{\zeta})\right. \\
& \left.\quad+\left(\partial_{r} \psi+\frac{\psi}{r}\right)\left(\partial_{z} \bar{v}\right)\right]+\left(f+\frac{2 \bar{v}}{r}\right)\left[\left(\partial_{z}^{2} \psi\right)(f+\bar{\zeta})+\left(\partial_{z} \psi\right)\left(\partial_{z} \bar{\zeta}\right)-\left(\partial_{r z}^{2} \psi+\frac{\partial_{z} \psi}{r}\right)\left(\partial_{z} \bar{v}\right)-\left(\partial_{r} \psi+\frac{\psi}{r}\right)\left(\partial_{z}^{2} \bar{v}\right)\right]=0
\end{aligned}
$$

If we examine the limit of this expression as $r \rightarrow \infty$, assume constant shear and stratification (i.e., $\partial_{r} \bar{b}, \partial_{z} \bar{b}, \partial_{r} \bar{v}$, and $\partial_{z} \bar{v}$ are constant), and replace the vertical shear using Eq. (A11), it is readily shown that Eq. (A15) reduces to the OoyamaHoskins equation valid under TWB:

$$
\partial_{t}^{2}\left(\partial_{z}^{2} \psi+\partial_{r}^{2} \psi\right)+\left(N^{2} \partial_{r}^{2} \psi-2 M^{2} \partial_{r z}^{2} \psi+F^{2} \partial_{z}^{2} \psi\right)=0 .
$$

To facilitate comparison with Eq. (19) of Ooyama (1966) or the first equation of Hoskins (1974), in the above expression we have defined $M^{2}=\partial_{r} \bar{b}, N^{2}=\partial_{z} \bar{b}$, and $F^{2}=f(f+\bar{\zeta})$. We return to this simplified model below in our discussion of a more general model for flows in GWB (section e of appendix A).

\section{b. Ellipticity and hyperbolicity}

While a general solution to Eq. (A15) is not evident to the present authors (see also Ooyama 1966; Kloosterziel 2010), progress can nevertheless be made by examining the discriminant of the equation, which depends on the basic (mean) state. Assuming solutions of the form $\psi=\Psi(r, z) e^{\sigma t}$, and restricting our solution to the steady state (i.e., $\partial_{t}^{2} \rightarrow \sigma^{2}=0$ ), we obtain the following elliptic and hyperbolic conditions. The discriminant of the PDE is $\Delta=B^{2}-A C$, with $A=N^{2}$, $B=-2(f+2 \bar{v} / r) \partial_{z} \bar{v}$, and $C=(f+\bar{\zeta})(f+2 \bar{v} / r)$. Thus, the PDE is elliptic when $\Delta<0$ and hyperbolic when $\Delta>0$. The latter is of interest, giving

$$
\Phi=(f+\bar{\zeta})\left(f+\frac{2 \bar{v}}{r}\right) N^{2}-\left(f+\frac{2 \bar{v}}{r}\right)^{2}\left|\partial_{z} \bar{v}\right|^{2}<0 .
$$

Expressed in isopycnal coordinates, for which $\partial_{r} \bar{b}=\partial_{z} \bar{v}=0$ and $N^{2}=\partial_{z} \bar{b}>0$, the criterion is

$$
\Phi=[(f+2 \bar{v} / r)(f+\bar{\zeta})]_{\rho}<0,
$$

where the expression in square brackets is evaluated along constant density surfaces. This matches the historical criterion attributed to Solberg (1936) and proved sufficient by Fjortoft (1950) and Ooyama (1966). We refer the reader to more recent discussions of this criterion by Cho et al. (1993), Kloosterziel (2010), and Yim et al. (2019).

\section{c. $P V$, absolute angular momentum, and additional forms of the criterion}

It is helpful to manipulate Eq. (A17) in order to see an explicit connection to the Ertel PV and absolute angular momentum. To understand how Eq. (A17) relates to the Ertel PV, we first define a signed, nondimensional number as $\mathrm{Cu}=2 \bar{v} /(f r)$, referred to in the main text as the curvature number. We then express the Ertel PV for a front in GWB as

$$
q=(f+\bar{\zeta}) N^{2}-\left(f+\frac{2 \bar{v}}{r}\right)\left|\partial_{z} \bar{v}\right|^{2}
$$


allowing us to rewrite Eq. (A17) as

$$
\Phi=(1+\mathrm{Cu}) f q<0 .
$$

Written in this form, it is immediately obvious that it is a generalization of Hoskins's criterion. For fronts in TWB, the criterion reduces to $\Phi=f q<0$.

The additional constraint on the curvature number $\mathrm{Cu}$ can be shown to result for axisymmetric flows, only. Moreover, it can be shown to result directly from conservation of absolute angular momentum. To see this, one first expresses the azimuthal momentum equation [cf. Eq. (A2) with $\partial_{\theta} \rightarrow 0$ ] as

$$
\partial_{t} v+u \partial_{r} v+\frac{u v}{r}+w \partial_{z} v+f u=0
$$

Following Rayleigh (1917), we note that this can be rewritten as

$$
\left(\partial_{t}+u \partial_{r}+w \partial_{z} v\right) L=D L / D t=0,
$$

where $L=r v+f r^{2} / 2$ is the absolute angular momentum on an $f$ plane (Holton 1992). Were gradients present in the azimuthal direction, this expression would not hold. For example, a pressure gradient on the right-hand side of Eq. (A21) would imply that $D L / D t \neq 0$. To what extent this criterion is valid, then, in the presence of baroclinic instability (Charney 1947; Eady 1949) is unknown to the authors. A study by Billant and Gallaire (2005) may shed light on this question.

Given this definition of $L$, one can alternatively express the curvature number in terms of $L$ as $1+\mathrm{Cu}=2 L /\left(\mathrm{fr}^{2}\right)$. This motivates the following form of the instability criterion:

$$
\Phi=\frac{2 L q}{r^{2}}<0
$$

(Kloosterziel et al. 2007), or simply

$$
L q<0 \text {. }
$$

Thus, both PV and absolute angular momentum play governing roles in the stability of the axisymmetric vortex. If one of these (but not both) is negative, we can anticipate instability. If both are negative, the product will be positive, corresponding to a stable, intense anticyclone that is a geophysically unlikely but theoretically possible scenario.

\section{d. Connection to Rayleigh (1917)}

Rayleigh has stated that the fluid "is stable only under the condition that the circulation $[K=r v]$ always increases with $r$ ", (Rayleigh 1917; Chandrasekhar 1961), where the text in brackets has been added for clarity. In replacing Rayleigh's circulation $K$ with absolute angular momentum $L$, one finds that a necessary and sufficient criterion for inertial instability is

$$
\frac{1}{r^{3}} \frac{d}{d r}\left(L^{2}\right)=\chi^{2}=(f+2 \bar{v} / r)(f+\bar{\zeta})<0 .
$$

(Kloosterziel et al. 2007). Together with Eq. (A18), this demonstrates that if the gradient of squared absolute angular momentum $L^{2}$ is taken along an isopycnal and decreases with increasing distance from the vortex center, the flow is unstable. This implies that isopycnals inclined to surfaces of absolute angular momentum are an indication of instability, a metric often used in meteorology (Holton 1992).

\section{e. A limiting solution}

While the foregoing arguments make the generalized Rayleigh criterion a very likely one, one should still like to establish its sufficiency directly from the governing perturbation equation. Here, we have borrowed words from Chandrasekhar (1961, chapter 7, section 66) in his discussion of Rayleigh's barotropic criterion. Below, we do this for the case of symmetric instability.

Eigen- or normal-mode solutions to Eq. (A15) can be obtained for limiting cases (Yanai 1961; Nelson et al. 2013; Brannigan et al. 2017). For cases in which the gradients of density and velocity can be considered to vary slowly over the scale of perturbations, one can use the Wentzel-Kramers-Brillouin approximation to obtain an expression for the growth rate of the disturbance. This has been done, for instance, by Brannigan et al. (2017, their appendix (C), where they approximate the solution at $r=r_{o}$. For parcel motions aligned with isopycnals - that is, describing the fastest-growing mode - their expression simplifies to $\sigma^{2} / f^{2}=(1+$ $\mathrm{Cu})^{2} \mathrm{Ri}^{-1}-(1+\mathrm{Cu})(1+\mathrm{Ro})=-\Phi^{\prime}$, demonstrating that 1$)$ instability occurs for $\Phi^{\prime}<0$, giving positive growth rates, and 2) energetic disturbances within symmetric instability have growth rates that scale as $\sigma^{2} \sim f^{2} \Phi^{\prime}$, where $\Phi^{\prime}$ is a nondimensional generalized Rayleigh discriminant. Thus, $\Phi^{\prime}<0$ is a necessary and sufficient criterion for instability. In this expression, $\operatorname{Ro}=\bar{\zeta} / f$, $\mathrm{Ri}=N^{2} /\left|\partial_{z} \bar{v}\right|^{2}$, and $\mathrm{Cu}$ has been defined above.

This same result can be obtained directly from the governing equation [cf. Eq. (A15)]. One simply 1) approximates all gradients in velocity and buoyancy as constant near a radius $r_{o}$ sufficiently far from the vortex center, 2) assumes a normal-mode solution $\psi=\Psi_{o} e^{\sigma t} e^{i(k r+m z)}$, where $k$ and $m$ are horizontal and vertical wavenumbers, and 3 ) requires that the vertical scale of the disturbance be much smaller than the radius $r_{o}$, or $1 /\left(m r_{o}\right) \ll 1$. We find this results in a simple analytical frontal model and dispersion relation, despite that it only applies for large $m r_{o}$.

Conducting the above steps leads to a frontal model that is comparable to the Ooyama-Hoskins model [cf. Eq. (A16)] except that $F^{2}=f(f+\bar{\zeta})$ is replaced by the barotropic Rayleigh discriminant $\chi^{2}=(f+2 \bar{v} / r)(f+\bar{\zeta})$ and $M^{2}=\partial_{r} \bar{b}$ implicitly contains curvature effects [cf. Eq. (A11)]:

$$
\partial_{t}^{2}\left(\partial_{z}^{2} \psi+\partial_{r}^{2} \psi\right)+\left(N^{2} \partial_{r}^{2} \psi-2 M^{2} \partial_{r z}^{2} \psi+\chi^{2} \partial_{z}^{2} \psi\right)=0 .
$$

Introducing the modal form $\psi$ and defining $\tau=\tan \beta=k / m$, where $-\beta$ defines the angle that the velocity vector $\left(u^{\prime}, w^{\prime}\right)$ makes with the horizontal plane (Hoskins 1974; Thomas et al. 2013), we obtain the following dispersion relation:

$$
\frac{\sigma^{2}}{f^{2}}=-\cos ^{2} \beta\left(\frac{N^{2} \tau^{2}}{f^{2}}-\frac{2 M^{2} \tau}{f^{2}}+\frac{\chi^{2}}{f^{2}}\right),
$$

where $\cos ^{2} \beta=\left(1+\tau^{2}\right)^{-1}$. As was done by Hoskins (1974), we can then define a variable-say, $\Phi=\chi^{2} N^{2}-M^{4}$ - that can be shown to be minus the discriminant of the quadratic expression, placing constraints on the sign of $\Phi$ for growing disturbances $\left(\sigma^{2}>0\right)$. In addition, by rearranging the quadratic expression in parentheses (e.g., Bachman and Taylor 2014; Brannigan et al. 2017), one can rewrite the dispersion relation as 


$$
\frac{\sigma^{2}}{f^{2}}=-\cos ^{2} \beta\left[\frac{N^{2}}{f^{2}}\left(\tau-\frac{M^{2}}{N^{2}}\right)^{2}+\Phi^{\prime}\right],
$$

where $\Phi^{\prime}=\chi^{2} / f^{2}-M^{4} /\left(f^{2} N^{2}\right)$ is the nondimensional Rayleigh discriminant defined above. For modes aligned with isopycnals, $\tau=k / m=M^{2} / N^{2}$, the dimensional dispersion relation simplifies to $\sigma^{2}=-f^{2} \Phi^{\prime} \cos ^{2} \beta$. Since $\cos ^{2} \beta>0$ for all $\beta$, this demonstrates that 1) $\Phi^{\prime}$ must be negative for growing disturbances and 2) disturbances under symmetric instability grow at a squared rate that scales as $-f^{2} \Phi^{\prime}$.

Given this model and normal-mode solution, is possible to obtain expressions for energy sources in the perturbation or turbulent kinetic energy equation (e.g., Smyth and McWilliams 1998; Brannigan et al. 2017) using Eqs. (A6)-(A9). This was done, for example, for the case of TWB by Thomas et al. (2013). Given the restrictions inherent in our model [cf. Eq. (A26)] and our uncertainty with regard to its applicability to submesoscale fronts and vortices, we leave this as an exercise for the reader or will consider this ourselves in a future study. For now, we simply note that a limiting normal-mode solution is possible and that, for symmetric instability, the squared growth rates of disturbances scale as $\sigma^{2} \sim$ $-f^{2} \Phi^{\prime}$, where $\Phi^{\prime}=(1+\mathrm{Cu})(1+\mathrm{Ro})-(1+\mathrm{Cu})^{-2} \mathrm{Ri}^{-1}$ is a nondimensional form of the Rayleigh discriminant.

\section{APPENDIX B}

\section{Instability Categories (GI, CI, SI) for Curved Density Fronts}

Thomas et al. (2013) introduced a method of categorizing instability types using nondimensional numbers, Ro and $\mathrm{Ri}$, and their effect on the Ertel PV. It is a visually powerful technique because it allows one to gauge in a graphical manner what drives the discriminant to have opposite sign and, thus, is an indication of instability. This should be qualified because-at least in observations-one might simply be seeing the result of an instability that has already occurred. In light of the present study, where curvature plays an important role, it appears helpful to modify these instability categories.

The categorization of instabilities depends upon modified gradient Rossby and Richardson numbers (Thomas et al. 2013). These values express the relative roles of horizontal shear, stratification, and vertical shear in modifying the Ertel PV (Ertel 1942). See, also, Li et al. (2012) and Hamlington et al. (2014) for cases in which surface wave effects are present. As in the main text [cf. Eq. (14)], the nondimensional Ertel PV under gradient wind balance $(\mathrm{GWB})$ is

$$
q^{\prime}=1+\mathrm{Ro}-(1+\mathrm{Cu}) \mathrm{Ri}^{-1}
$$

For clarity, we again define gradient Rossby, Richardson, and curvature numbers as

$$
\begin{aligned}
& \mathrm{Ro}=\bar{\zeta} / f, \\
& \mathrm{Ri}=\frac{N^{2}}{\left|\partial_{z} \bar{v}\right|^{2}}, \\
& \mathrm{Cu}=\frac{2 \bar{v}}{f R} .
\end{aligned}
$$

In Eqs. (B2)-(B4), $\bar{v}$ is mean alongfront component of the flow and includes both geostrophic and ageostrophic velocities. Also, note that we have replaced the radius of a vortex $r$ with a local radius of curvature $R$ to illustrate more clearly how the expressions might be applied to both curved fronts and vortices (cf. Fig. 2). For curved fronts, the mean flow is no longer a signed quantity, so that $R$ must be signed-that is, positive for cyclonic flow and negative for anticyclonic flow [cf. Eq. (B7)].

For curved fronts with symmetry in the direction of the gradient wind shear, the relevant discriminant is not the Ertel PV but the product of the Ertel PV and absolute angular momentum [cf. Eq. (17)]:

$$
\Phi^{\prime}=L^{\prime} q^{\prime}=\underbrace{(1+\mathrm{Cu})(1+\mathrm{Ro})}_{\text {barotropic component }}-\underbrace{(1+\mathrm{Cu})^{2} \mathrm{Ri}^{-1}}_{\text {baroclinic component }}<0 .
$$

where $L^{\prime}=1+\mathrm{Cu}$ is the nondimensional absolute angular momentum. The first term in Eq. (B5) contains information about inertial or centrifugal instability, and the second term contains information about gravitational and symmetric instability, both of which are amplified in low-stratified environments.

Given this interpretation, it is simple to modify the instability categories of Thomas et al. (2013):

$$
\begin{aligned}
\phi_{\mathrm{Ro}} & \rightarrow \phi_{o}=\tan ^{-1}[-(1+\mathrm{Cu})(1+\mathrm{Ro})] \\
\phi_{\mathrm{Ri}} & \rightarrow \phi_{1}=\tan ^{-1}\left[-(1+\mathrm{Cu})^{2} \mathrm{Ri}^{-1}\right],
\end{aligned}
$$

where $\phi_{\mathrm{Ro}}$ and $\phi_{\mathrm{Ri}}$ are the barotropic and baroclinic angles previously defined by Thomas et al. (2013). As noted by Hamlington et al. (2014), the inverse tangent effectively compresses the dynamically relevant information contained in Ro, $\mathrm{Ri}$, and $\mathrm{Cu}$ into a smaller range of values. To determine the instability type, one would estimate $\mathrm{Ro}, \mathrm{Ri}$, and $\mathrm{Cu}$ within different parts of the front or vortex, compute the angles $\phi_{o}$ and $\phi_{1}$ and then classify the flow. Note that the expressions in Eq. (B6) reduce to those of Thomas et al. (2013) in the limit $\mathrm{Cu} \rightarrow 0$ so that these are valid for both straight and curved fronts. The possible instabilities include gravitational instability (GI), symmetric instability (SI), and pure inertial or centrifugal instability (CI), and the classification of these instability types from the above angles is given in Table B1 (see also Hamlington et al. 2014).

Note that the instability classification given above is not interpreted in terms of energy exchange under linear instability. Rather, it should be interpreted as an answer to the question, "What aspect of the flow is most responsible for reducing the stability discriminant, $\Phi^{\prime}$ ?" In this manner, our definition differs slightly from that given by Thomas et al. (2013), though we note that the growth rates of disturbances do scale with $\Phi^{\prime}$ for parcel motions aligned with isopycnals - that is, symmetric instability (section e of appendix A). We are presently investigating application of these instability categories to observations and model simulations.

\section{Radius of curvature}

To help the reader to apply this expression to curved fronts that might not have a distinct radius, we note that it is straightforward to calculate the radius of curvature, $R$, along 
TABLE B1. Frontal instability categories from gradient Rossby and Richardson numbers when accounting for curvature. Instability types include gravitational instability (GI), symmetric instability (SI), and centrifugal instability (CI). Definitions for $\phi_{o}$ and $\phi_{1}$ are given in Eq. (B6).

\begin{tabular}{ccc}
\hline \hline Instability category & Cyclonic & Anticyclonic \\
\hline Stable & $\phi_{o}<\phi_{1} \leq 0$ & $\phi_{o}<\phi_{1} \leq 0$ \\
CI/SI & - & $-\pi / 4<\phi_{1} \leq \phi_{o}$ \\
SI & $-\pi / 2<\phi_{1} \leq \phi_{o}$ & $-\pi / 2<\phi_{1} \leq-\pi / 4$ \\
SI/GI & $-3 \pi / 4<\phi_{1} \leq-\pi / 2$ & $-3 \pi / 4<\phi_{1} \leq-\pi / 2$ \\
GI & $-\pi<\phi_{1} \leq-3 \pi / 4$ & $-\pi<\phi_{1} \leq-3 \pi / 4$ \\
\hline
\end{tabular}

smooth contours, such as dynamic height or a frontal boundary separating different sea surface temperatures. One simply estimates the radius of curvature as $R=1 / \kappa$, where

$$
\kappa=\frac{\dot{x} \ddot{y}-\dot{y} \ddot{x}}{\left(\dot{x}^{2}+\dot{y}^{2}\right)^{3 / 2}} .
$$

is the geometric curvature, and $\dot{x}, \dot{y}, \ddot{x}$, and $\ddot{y}$ denote first and second derivatives of zonal and meridional displacements with respect to distance along the boundary $s$-for example, $\dot{x}=d x / d s$ (cf. Fig. 2). We note, however, that one must ensure smooth contours to obtain a realistic locally valid estimate of $R$. In this sense, it is somewhat subjective, but minimal smoothing produces reasonable estimates (Buckingham et al. 2017, their appendix A).

\section{REFERENCES}

Adams, K. A., P. Hosegood, J. R. Taylor, J.-B. Sallée, S. Bachman, R. Torres, and M. Stamper, 2017: Frontal circulation and submesoscale variability during the formation of a Southern Ocean mesoscale eddy. J. Phys. Oceanogr., 47, 1737-1753, https://doi.org/10.1175/JPO-D-16-0266.1.

Bachman, S. D., and J. R. Taylor, 2014: Modelling of partiallyresolved oceanic symmetric instability. Ocean Modell., 82, 15-27, https://doi.org/10.1016/j.ocemod.2014.07.006.

—_, B. Fox-Kemper, J. R. Taylor, and L. N. Thomas, 2017: Parameterization of frontal symmetric instabilities. Part I: Theory for resolved fronts. Ocean Modell., 109, 72-95, https:// doi.org/10.1016/j.ocemod.2016.12.003.

Billant, P., and F. Gallaire, 2005: Generalized Rayleigh criterion for non-axisymmetric centrifugal instabilities. J. Fluid Mech., 542, 365-379, https://doi.org/10.1017/S0022112005006464.

Brannigan, L., D. P. Marshall, A. C. Naveira Garabato, A. J. G. Nurser, and J. Kaiser, 2017: Submesoscale instabilities in mesoscale eddies. J. Phys. Oceanogr., 47, 3061-3085, https:// doi.org/10.1175/JPO-D-16-0178.1.

Buckingham, C. E., Z. Khaleel, A. Lazar, A. P. Martin, J. T. Allen, A. C. Naveira Garabato, A. F. Thompson, and C. Vic, 2017: Testing Munk's hypothesis for submesoscale eddy generation using observations in the North Atlantic. J. Geophys. Res. Oceans, 122, 6725-6745, https://doi.org/10.1002/ 2017JC012910.

_- N. Lucas, S. E. Belcher, T. Rippeth, A. Grant, J. Le Sommer, A. O. Ajayi, and A. Naveira Garabato, 2019: The contribution of surface and submesoscale processes to turbulence in the open ocean surface boundary layer. J. Adv. Model. Earth Syst., 11, 4066-4094, https://doi.org/ 10.1029/2019MS001801.
_ J. Gula, and X. Carton, 2021: The role of curvature in modifying frontal instabilities. Part II: Application of the criterion to curved density fronts at low Richardson numbers. J. Phys. Oceanogr., 51, 317-341, https://doi.org/10.1175/JPO-D-20-0258.1.

Chandrasekhar, S., 1961: Hydrodynamic and Hydromagnetic Stability. Dover, $652 \mathrm{pp}$.

Charney, J. G., 1947: The dynamics of long waves in a baroclinic westerly current. J. Meteor., 4, 136-162, https://doi.org/10.1175/ 1520-0469(1947)004<0136:TDOLWI >2.0.CO;2.

Cho, H.-R., T. G. Shepherd, and V. A. Vladimirov, 1993: Application of the direct Liapunov method to the problem of symmetric stability in the atmosphere. J. Atmos. Sci., 50, 822-836, https:// doi.org/10.1175/1520-0469(1993)050<0822:AOTDLM>2.0.CO;2.

Colin de Verdière, A., 2012: The stability of short symmetric internal waves on sloping fronts: Beyond the traditional approximation. J. Phys. Oceanogr., 42, 459-475, https://doi.org/10.1175/JPO-D11-067.1.

Cornillon, P. C., E. Firing, A. F. Thompson, L. M. Ivanov, I. Kamenkovich, C. E. Buckingham, and Y. D. Afanasyev, 2019: Oceans. Zonal Jets: Phenomenology, Genesis, and Physics, B. Galperin and P. L. Read, Eds., Cambridge University Press, 46-71, https://doi.org/10.1017/9781107358225.003.

D’Asaro, E., C. Lee, L. Rainville, R. Harcourt, and L. Thomas, 2011: Enhanced turbulence and energy dissipation at ocean fronts. Science, 332, 318-322, https://doi.org/10.1126/science.1201515.

Dee, D. P., and Coauthors, 2011: The ERA-Interim reanalysis: Configuration and performance of the data assimilation system. Quart. J. Roy. Meteor. Soc., 137, 553-597, https://doi.org/ 10.1002/qj.828.

Deremble, B., N. Wienders, and W. K. Dewar, 2014: Potential vorticity budgets in the North Atlantic Ocean. J. Phys. Oceanogr., 44, 164-178, https://doi.org/10.1175/JPO-D-13-087.1.

Dong, S., J. Sprintall, and S. T. Gille, 2006: Location of the Antarctic polar front from AMSR-E satellite sea surface temperature measurements. J. Phys. Oceanogr., 36, 2075-2089, https://doi.org/ 10.1175/JPO2973.1.

Eady, E. T., 1949: Long waves and cyclone waves. Tellus, 1, 33-52, https://doi.org/10.1111/j.2153-3490.1949.tb01265.x.

Eden, C., and J. Willebrand, 1999: Neutral density revisited. Deep-Sea Res. II, 46, 33-54, https://doi.org/10.1016/S0967-0645(98)00113-1.

Eliassen, A., 1951: Slow thermally or frictionally controlled meridional circulation in a circular vortex. Astrophys. Norv., 5, 19-60.

— 1962: On the vertical circulation in frontal zones. Geofys. Publ., 24, 147-160.

—, and E. Kleinschmidt Jr., 1957: Dynamic meteorology. Handbuch der Physik, Springer-Verlag, 1-154.

Ertel, H., 1942: Ein neuer hydrodynamischer wirbelsatz. Meteor. Z., 59, 271-281.

Fjortoft, R., 1944: On the frontogenesis and cyclogenesis in the atmosphere. Part I. On the stability of the stationary circular vortex. Geofys. Publ., 16, 1-28.

- 1950: Application of integral theorems in deriving criteria of stability for laminar flows and for the baroclinic circular vortex. Geophys. Publ., 17, 1-52.

Fox-Kemper, B., and Coauthors, 2011: Parameterization of mixed layer eddies. III: Implementation and impact in global ocean climate simulations. Ocean Modell., 39, 61-78, https://doi.org/ 10.1016/j.ocemod.2010.09.002.

Gill, A. E., 1982: Atmosphere-Ocean Dynamics. Academic Press, $662 \mathrm{pp}$.

Grooms, I., 2015: Submesoscale baroclinic instability in the balance equations. J. Fluid Mech., 762, 256-272, https://doi.org/10.1017/ jfm.2014.657. 
Gula, J., T. M. Blacic, and R. E. Todd, 2019: Submesoscale coherent vortices in the Gulf Stream. Geophys. Res. Lett., 46, 2704-2714, https://doi.org/10.1029/2019GL081919.

Hamlington, P. E., L. P. V. Roekel, B. Fox-Kemper, K. Julien, and G. P. Chini, 2014: Langmuir-submesoscale interactions: Descriptive analysis of multiscale frontal spindown simulations. J. Phys. Oceanogr., 44, 2249-2272, https://doi.org/10.1175/JPOD-13-0139.1.

Haynes, P. H., and M. E. McIntyre, 1987: On the evolution of vorticity and potential vorticity in the presence of diabatic heating and frictional or other forces. J. Atmos. Sci., 44, 828-841, https:// doi.org/10.1175/1520-0469(1987)044〈0828:OTEOVA〉2.0.CO;2.

Holmes, R. M., L. N. Thomas, L. Thompson, and D. Darr, 2014: Potential vorticity dynamics of tropical instability vortices. J. Phys. Oceanogr., 44, 995-1011, https://doi.org/10.1175/JPOD-13-0157.1.

Holton, J. R., 1992: An Introduction to Dynamic Meteorology. 3rd ed. Academic Press, 507 pp.

Hoskins, B., 2015: Potential vorticity and the PV perspective. $A d v$. Atmos. Sci., 32, 2-9, https://doi.org/10.1007/s00376-014-0007-8.

Hoskins, B. J., 1974: The role of potential vorticity in symmetric stability and instability. Quart. J. Roy. Meteor. Soc., 100, 480482, https://doi.org/10.1002/qj.49710042520.

— models: Mathematical formulation and solution. J. Atmos. Sci., 29, 11-37, https://doi.org/10.1175/1520-0469(1972)029<0011: AFMMFA $>2.0 . \mathrm{CO} ; 2$.

—, M. E. McIntyre, and A. W. Robertson, 1985: On the use and significance of isentropic potential vorticity maps. Quart. J. Roy. Meteor. Soc., 111, 877-946, https://doi.org/10.1002/ qj.49711147002.

Jackett, D. R., and T. J. McDougall, 1997: A neutral density variable for the world's oceans. J. Phys. Oceanogr., 27, 237-263, https://doi.org/10.1175/1520-0485(1997)027<0237:ANDVFT> 2.0.CO;2.

Klein, P., and G. Lapeyre, 2009: The oceanic vertical pump induced by mesoscale and submesoscale turbulence. Annu. Rev. Mar. Sci., 1, 351-375, https://doi.org/10.1146/annurev.marine.010908.163704.

Kloosterziel, R. C., 2010: Viscous symmetric stability of circular flows. J. Fluid Mech., 652, 171-193, https://doi.org/10.1017/ S0022112009994149.

_ _ and G. J. F. van Heijst, 1991: An experimental study of unstable barotropic vortices in a rotating fluid. J. Fluid Mech., 223, 1-24, https://doi.org/10.1017/S0022112091001301.

_ , G. F. Carnevale, and P. Orlandi, 2007: Inertial instability in rotating and stratified fluids: Barotropic vortices. J. Fluid Mech., 583, 379-412, https://doi.org/10.1017/S0022112007006325.

Kreyszig, E., 1998: Advanced Engineering Mathematics. 8th ed. John Wiley and Sons, $1288 \mathrm{pp}$.

Kundu, P. K., and I. M. Cohen, 2008: Fluid Mechanics. 4th ed. Elsevier, 904 pp.

Lamb, S. H., 1932: Hydrodynamics. 6th ed. Cambridge University Press, $738 \mathrm{pp}$.

Lazar, A., A. Stegner, and E. Heifetz, 2013: Inertial instability of intense stratified anticyclones. Part I. generalized stability criterion. J. Fluid Mech., 732, 457-484, https://doi.org/10.1017/ jfm.2013.412.

Lévy, M., R. Ferrari, P. J. S. Franks, A. P. Martin, and P. Riviére, 2012: Bringing physics to life at the submesoscale. Geophys. Res. Lett., 39, L14602, https://doi.org/10.1029/2012GL052756.

Li, K., Z. Zhang, G. Chini, and G. Flierl, 2012: Langmuir circulation: An agent for vertical restratification? J. Phys. Oceanogr., 42, 1945-1958, https://doi.org/10.1175/JPO-D-11-0225.1.
Li, Q., X. Mao, J. Huthnance, S. Cai, and S. Kelly, 2019: On internal waves propagating across a geostrophic front. J. Phys. Oceanogr., 49, 1229-1248, https://doi.org/10.1175/JPO-D-18-0056.1.

Mahadevan, A., 2016: The impact of submesoscale physics on primary productivity of plankton. Annu. Rev. Mar. Sci., 8, 161184, https://doi.org/10.1146/annurev-marine-010814-015912.

Marshall, J. C., and A. J. G. Nurser, 1992: Fluid dynamics of oceanic thermocline ventilation. J. Phys. Oceanogr., 22, 583-595, https://doi.org/10.1175/1520-0485(1992)022<0583:FDOOTV> 2.0.CO;2.

McDougall, T. J., 1987: Neutral surfaces. J. Phys. Oceanogr., 17, 1950-1964, https://doi.org/10.1175/1520-0485(1987)017<1950: NS>2.0.CO;2.

McWilliams, J. C., 1985: Submesoscale, coherent vortices in the ocean. Rev. Geophys., 23, 165-182, https://doi.org/10.1029/ RG023i002p00165.

- 2016: Submesoscale currents in the ocean. Proc. Roy. Soc. London, 472A, 1-32, https://doi.org/10.1098/rspa.2016.0117.

Mooers, C. N. K., 1975: Several effects of baroclinic currents on the three-dimensional propagation of inertial-internal waves. Geophys. Fluid Dyn., 6, 277-284, https://doi.org/10.1080/ 03091927509365798.

Morel, Y., J. Gula, and A. Ponte, 2019: Potential vorticity diagnostics based on balances between volume integral and boundary conditions. Ocean Modell., 138, 23-35, https://doi.org/10.1016/ j.ocemod.2019.04.004.

Müller, P., 1995: Ertel's potential vorticity theorem in physical oceanography. Rev. Geophys., 33, 67-97, https://doi.org/10.1029/ 94RG03215.

Mutabazi, I., C. Normand, and J. E. Wesfreid, 1992: Gap size effects on centrifugally and rotationally driven instabilities. Phys. Fluids, 4A, 1199-1205, https://doi.org/10.1063/ 1.858238.

Nelson, R. P., O. Gressel, and O. M. Umurhan, 2013: Linear and non-linear evolution of the vertical shear instability in accretion discs. Mon. Not. Roy. Astron. Soc., 435, 2610-2632, https:// doi.org/10.1093/mnras/stt1475.

Ooyama, K., 1966: On the stability of the baroclinic circular vortex: A sufficient criterion for instability. J. Atmos. Sci., 23, 43-53, https:// doi.org/10.1175/1520-0469(1966)023<0043:OTSOTB >2.0.CO;2.

Pedlosky, J., 1987: Geophysical Fluid Dynamics. 2nd ed. SpringerVerlag, $710 \mathrm{pp}$.

Rayleigh, L., 1917: On the dynamics of revolving fluids. Proc. Roy. Soc. London, 93, 148-154, https://doi.org/10.1098/rspa.1917.0010.

Rüdiger, G., R. Arlt, and D. Shalybkov, 2002: Hydrodynamic stability in accretion disks under the combined influence of shear and density stratification. Astron. Astrophys., 391, 781-787, https://doi.org/10.1051/0004-6361:20020853.

Sawyer, J. S., 1956: The vertical circulation at meteorological fronts and its relation to frontogenesis. Proc. Roy. Soc. London, 234A, 346-362, https://doi.org/10.1098/rspa.1956.0039.

Shakespeare, C. J., 2016: Curved density fronts: Cyclogeostrophic adjustment and frontogenesis. J. Phys. Oceanogr., 46, 31933207, https://doi.org/10.1175/JPO-D-16-0137.1.

Shapiro, L. J., and H. E. Willoughby, 1982: The response of balanced hurricanes to local sources of heat and momentum. J. Atmos. Sci., 39, 378-394, https://doi.org/10.1175/1520-0469(1982)039<0378: TROBHT $>2.0 . \mathrm{CO} ; 2$.

Smith, K. M., P. E. Hamlington, and B. Fox-Kemper, 2016: Effects of submesoscale turbulence on ocean tracers. J. Geophys. Res. Oceans, 121, 908-933, https://doi.org/10.1002/2015JC011089.

Smyth, W. D., and J. C. McWilliams, 1998: Instability of an axisymmetric vortex in a stably stratified, rotating environment. 
Theor. Comput. Fluid Dyn., 11, 305-322, https://doi.org/10.1007/ s001620050095.

Sokolov, S., and S. R. Rintoul, 2009: Circumpolar structure and distribution of the Antarctic circumpolar current fronts: 1. Mean circumpolar paths. J. Geophys. Res., 114, C11018, https://doi.org/ 10.1029/2008JC005108.

Solberg, H., 1936: Le mouvement d'inertie de l'atmosphere stable et son role dans la theorie des cyclones. Sixth Assembly, Edinburgh, United Kingdom, Union Geodesique et Geophysique Internationale, 66-82.

Spall, M. A., 1995: Frontogenesis, subduction, and cross-front exchange at upper ocean fronts. J. Geophys. Res., 100, 2543-2557, https://doi.org/10.1029/94JC02860.

Straub, D. N., 1999: On thermobaric production of potential vorticity in the ocean. Tellus, 51A, 314-325, https://doi.org/10.3402/ tellusa.v51i2.12313.

Taylor, G. I., 1923: Stability of a viscous liquid contained between two rotating cylinders. Philos. Trans. Roy. Soc. London, 223, 289-343, https://doi.org/10.1098/rsta.1923.0008.

Taylor, J. R., and R. Ferrari, 2009: On the equilibration of a symmetrically unstable front via a secondary shear instability. J. Fluid Mech., 622, 103-113, https://doi.org/10.1017/ S0022112008005272.

— mixed layer density fronts. J. Phys. Oceanogr., 40, 1222-1242, https://doi.org/10.1175/2010JPO4365.1.

Thomas, L. N., 2008: Formation of intrathermocline eddies at ocean fronts by wind-driven destruction of potential vorticity. Dyn. Atmos. Oceans, 45, 252-273, https://doi.org/10.1016/ j.dynatmoce.2008.02.002.

_ , and J. R. Taylor, 2010: Reduction of the usable wind-work on the general circulation by forced symmetric instability.
Geophys. Res. Lett., 37, L18606, https://doi.org/10.1029/ 2010 GL044680.

—, A. Tandon, and A. Mahadevan, 2008: Submesoscale processes and dynamics. Ocean Modeling in an Eddying Regime, Geophys. Monogr., Vol. 177, Amer. Geophys. Union, 17-38.

_, J. R. Taylor, R. Ferrari, and T. M. Joyce, 2013: Symmetric instability in the Gulf Stream. Deep-Sea Res. II, 91, 96-110, https://doi.org/10.1016/j.dsr2.2013.02.025.

Thorpe, A. S., and R. Rotunno, 1989: Nonlinear aspects of symmetric instability. J. Atmos. Sci., 46, 1285-1299, https://doi.org/ 10.1175/1520-0469(1989)046<1285:NAOSI $>2.0 . C O ; 2$.

van Mieghem, J. M., H. R. Byers, H. E. Landsberg, H. Wexler, B. Haurwitz, A. F. Spilhaus, H. C. Willett, and H. G. Houghton, 1951: Hydrodynamic instability. Compendium of Meteorology, Amer. Meteor. Soc., 434-453, https://doi.org/10.1007/978-1940033-70-9_37.

Verdy, A., and M. R. Mazloff, 2017: A data assimilating model for estimating Southern Ocean biogeochemistry. J. Geophys. Res. Oceans, 122, 6968-6988, https://doi.org/10.1002/2016JC012650.

Wenegrat, J. O., and L. N. Thomas, 2017: Ekman transport in balanced fronts with curvature. J. Phys. Oceanogr., 47, 1189-1203, https:// doi.org/10.1175/JPO-D-16-0239.1.

Yanai, M., 1961: Dynamical aspects of typhoon formation. J. Meteor. Soc. Japan, 39, 282-309, https://doi.org/10.2151/jmsj1923.39.5_282.

_ in the baroclinic circular vortex: A numerical experiment. J. Meteor. Soc. Japan, 47, 183-198, https://doi.org/10.2151/ jmsj1965.47.3_183.

Yim, E., A. Stegner, and P. Billant, 2019: Stability criterion for the centrifugal instability of surface intensified anticyclones. J. Phys. Oceanogr., 49, 827-849, https://doi.org/10.1175/JPO-D18-0088.1. 